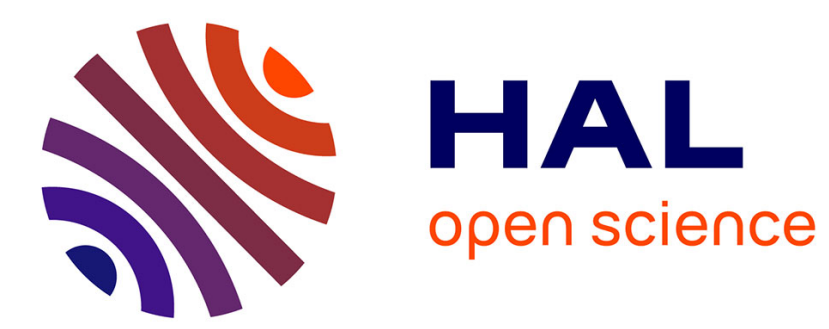

\title{
Slug genesis in cylindrical pipe flow
}

Y. Duguet, A.P. Willis, R.R. Kerswell

\section{- To cite this version:}

Y. Duguet, A.P. Willis, R.R. Kerswell. Slug genesis in cylindrical pipe flow. Journal of Fluid Mechanics, 2010, 663 (novembre), pp.180-208. 10.1017/s0022112010003435 . hal-01021118

\section{HAL Id: hal-01021118 \\ https://hal-polytechnique.archives-ouvertes.fr/hal-01021118}

Submitted on 16 Jul 2014

HAL is a multi-disciplinary open access archive for the deposit and dissemination of scientific research documents, whether they are published or not. The documents may come from teaching and research institutions in France or abroad, or from public or private research centers.
L'archive ouverte pluridisciplinaire HAL, est destinée au dépôt et à la diffusion de documents scientifiques de niveau recherche, publiés ou non, émanant des établissements d'enseignement et de recherche français ou étrangers, des laboratoires publics ou privés. 


\title{
Slug genesis in cylindrical pipe flow
}

\author{
Y. DUGUET, ${ }^{1,2,4} \dagger$ A. P. WILLIS ${ }^{1,3}$ AND R. R. KERSWELL ${ }^{1}$ \\ ${ }^{1}$ School of Mathematics, University of Bristol, BS8 1TW Bristol, UK \\ ${ }^{2}$ Linné Flow Centre, KTH Mechanics, SE-100 44 Stockholm, Sweden \\ ${ }^{3}$ Laboratoire d'Hydrodynamique, Ecole Polytechnique, 91128 Palaiseau, France \\ ${ }^{4}$ LIMSI-CNRS, UPR 3251, 91403 Orsay, France
}

(Received 19 January 2010; revised 24 June 2010; accepted 24 June 2010; first published online 5 October 2010)

\begin{abstract}
Transition to uniform turbulence in cylindrical pipe flow occurs experimentally via the spatial expansion of isolated coherent structures called 'slugs', triggered by localized finite-amplitude disturbances. We study this process numerically by examining the preferred route in phase space through which a critical disturbance initiates a 'slug'. This entails first identifying the relative attractor - 'edge state' - on the laminarturbulent boundary in a long pipe and then studying the dynamics along its lowdimensional unstable manifold, leading to the turbulent state. Even though the fully turbulent state delocalizes at $R e \approx 2300$, the edge state is found to be localized over the range $R e=2000-6000$, and progressively reduces in both energy and spatial extent as $R e$ is increased. A key process in the genesis of a slug is found to be vortex shedding via a Kelvin-Helmholtz mechanism from wall-attached shear layers quickly formed at the edge state's upstream boundary. Whether these shedded vortices travel on average faster or slower downstream than the developing turbulence determines whether a puff or a slug (respectively) is formed. This observation suggests that slugs are out-of-equilibrium puffs which therefore do not co-exist with stable puffs.
\end{abstract}

Key words: pipe-flow boundary layer, transition to turbulence, turbulence simulation

\section{Introduction}

The laminar flow of a Newtonian fluid inside a straight pipe of circular crosssection is known to undergo a transition to turbulence as the flow rate is increased, producing a dramatic rise in skin friction. Reynolds (1883) was the first to identify the 'Reynolds' number $R e:=U D / v$ as the sole controlling parameter for the flow, where $U$ is the bulk velocity, $D$ the diameter and $v$ the kinematic viscosity. Reynolds reported that the transition typically occurs for $R e \approx 2000$, a commonly quoted value in textbooks and widely reproduced ever since (critical $R e$ reported vary across the range 1760-2300, e.g. Binnie \& Fowler 1947, Lindgren 1958, Leite 1959, Wygnanski \& Champagne 1973 and Darbyshire \& Mullin 1995). Reynolds was also aware that if extra care was taken to reduce the amplitude of disturbances at the inlet, transition could be delayed (reportedly to $\approx 12000$ ), emphasizing the dependence of transition on the ambient noise level present and hence the inherently finite-amplitude nature of the process. By way of confirmation, Pfenniger (1961) managed to keep the flow laminar in a very well controlled experiment until $R e \approx 10^{5}$. 
When transition is triggered, turbulence first appears at isolated locations (not necessarily at the inlet), before expanding $(R e \gtrsim 2300)$ to contaminate the whole downstream domain (Reynolds 1883; Lindgren 1958, 1969; Wygnanski \& Champagne 1973; Mullin 2010). These growing patches of turbulent motion, which fill the whole cross-section of the pipe and are separated from each other by laminar regions, were called 'flashes' by Reynolds. Later a distinction was drawn between two different states christened 'puffs' and 'slugs' (Wygnanski \& Champagne 1973). Turbulent puffs correspond to a turbulent region with a sharp upstream boundary - the trailing edge (TE) - but whose downstream border - the leading edge (LE) - is much less well-defined, containing more large-scale structures which appear to decay away. They are observed experimentally for $R e \lesssim 2700$ (Wygnanski \& Champagne 1973) provided a large enough disturbance is applied to the flow, and for $R e \approx 1750-2200$, they tend to keep a constant length of about 20 pipe diameters as they propagate downstream. For $R e \gtrsim 2700$, slugs can be clearly identified in which the downstream boundary is reported to be as sharp as the upstream one. The enclosed turbulent motion, which fills the whole cross-section of the pipe, then looks indistinguishable from a fully turbulent pipe flow (Wygnanski \& Champagne 1973). The upstream front moves slower than the mean flow speed whereas the downstream moves faster, so that the slug grows in length as it is advected down the pipe (Lindgren 1969; Wygnanski \& Champagne 1973; Nishi et al. 2008). Little is known so far about the intermittent regime, intermediate between the puff and the slug regime. It is characterized by the disordered co-existence of laminar and turbulent domains in which puffs split and merge and possess sharpened slug-like LEs (Moxey \& Barkley 2010).

Given this background, two key questions are the following. What are the mechanisms by which puffs and slugs are formed? And what are the important processes which allow a puff to adopt an 'equilibrium' length but forces a slug to expand aggressively? Efforts to explain the observed behaviour initially concentrated on establishing the linear stability of the laminar Hagen-Poiseuille flow (Hagen $1839 \&$ Poiseuille 1840). Stability was proved under the assumption of axisymmetry (Herron 1991), and demonstrated in the general case up to $R e=10^{7}$ (Meseguer \& Trefethen 2003). At the other end of the spectrum, Joseph \& Carmi (1969) also proved that any disturbance would decay monotonically provided $R e<81.49$. The next logical step was to search for the emergence of alternative solutions between these two extremes, in the hope that this would make sense of the $O(2000)$ transition point. Despite some early success by Nagata (1990), who identified a steady-state solution in plane Couette flow, it was not until Waleffe $(1997,1998)$ uncovered the 'self-sustaining cycle' that it became clear how to find these new states. In a pipe flow, they take the form of travelling waves (TWs) (Faisst \& Eckhardt 2003; Wedin \& Kerswell 2004; Pringle \& Kerswell 2007; Pringle, Duguet \& Kerswell 2009). Experimental observations (Hof et al. 2004) and numerical experiments in short periodic pipes (Kerswell \& Tutty 2007; Schneider, Eckhardt \& Vollmer 2007a) demonstrated that these solutions appear fleetingly as coherent structures in pipe turbulence.

Work directed at establishing the role of these states in transition has tended to focus on the theoretical model of a short (typically $5 \mathrm{D}$ long) pipe along which periodic boundary conditions are imposed. Not only does this eliminate the issue of co-existence of turbulent- and laminar-flow regions, but it is much more efficient to simulate numerically. Following the work of Itano \& Toh (2001) in a plane Poiseuille flow, a key advance by Schneider, Eckhardt \& Yorke (2007b) was to realize that the laminar-turbulent boundary $\Sigma$ - the separatrix between the two local attractors could be traced numerically. By studying the dynamics restricted to this boundary, 
it became clear that 'lower branch' TWs are embedded within it (Duguet, Willis \& Kerswell 2008), so that initial conditions arbitrarily close to one of these TWs could either lead to turbulence or relaminarize (see e.g. Kerswell \& Tutty 2007, as well as Viswanath 2007 and Wang, Gibson \& Waleffe 2007 for plane Couette flow). Considerable progress has been made by viewing this restricted form of pipe flow and other shear flows as a huge dynamical system (see the review by Eckhardt et al. 2007 for a pipe flow, Viswanath 2007, Gibson, Halcrow \& Cvitanovic 2008, 2009 and Halcrow et al. 2009 for plane Couette flow).

The spatiotemporal dynamics of real pipe flows is, however, less clearly amenable to dynamical systems thinking as the flow is no longer in either the laminar or the turbulent state globally. As a result, our understanding is much more modest; yet only here is true contact possible with experimental observations. Fortunately, direct numerical simulation is now feasible in sufficiently long computational pipes ( $230 D$ long) to realistically capture 'puff' and 'slug' structures in pipe flow (Leonard \& Reynolds 1985; Shan et al. 1999; Priymak \& Miyazaki 2004; Reuter \& Rempfer 2004; Willis \& Kerswell 2007, 2008; Shimizu \& Kida 2008, 2009; Mellibovsky et al. 2009; Willis et al. 2009; Moxey \& Barkley 2010).

Very recent investigations (Shimizu \& Kida 2009; Hof et al. 2010) have proposed a mechanism for the sustenance of a turbulent puff, thereby opening the way for active control strategies. The suggested mechanism focuses on the role of local instabilities occurring upstream of the turbulent flow, triggering turbulent fluctuations which are convected downstream into the puff to presumably re-energize the vorticity field in the puff against slow viscous decay. These fluctuations initially take the form of rolled-up vortices emanating from the shear layer upstream of the puff (Bandhyopadyay 1986). Numerical simulation at higher $R e$ appears to be an adequate tool to investigate what becomes of this suggested mechanism in a non-equilibrium situation such as that of an expanding slug. The purpose of this paper is therefore to investigate how a slug is formed and to examine the relevance of this mechanism in the process. The key to this is identifying a natural starting point for detailed simulations. The edge state provides just such an initial condition because $(a)$ its unstable manifold directed across the laminar-turbulent boundary must lead to a slug being formed and $(b)$ arbitrary initial conditions close to and 'above' the threshold for triggering a slug will first shadow the laminar-turbulent boundary, being attracted towards the edge state, before being flung off along its unstable manifold (see the sketch in figure 1). Choosing to initiate our simulations in the neighbourhood of the edge state thus ensures that the evolution of the flow is least affected by the shape of an artificially prescribed initial condition. Following a flow trajectory starting very near the edge state also corresponds in phase space to the experimental situation when the amplitude of a disturbance is increased just enough to trigger turbulence. Therefore, our first (preliminary) objective is to compute the edge state at sufficiently high $\operatorname{Re}(\gtrsim 2700)$, which is appropriate for slugs to exist. Then the second (main) objective is to study how the flow metamorphoses into an expanding slug along the unstable manifold of the edge state.

This paper is organized as follows. In $\S 2$ we present the numerical tools used throughout the paper. Section 3 details the computation of edge states up to $R e=6000$, extending previous recent computations (Mellibovsky et al. 2009; Willis \& Kerswell 2009) which reported that the edge state stays localized even when the turbulent state is not. An interesting new observation made here is that the edge state becomes progressively smoother in both a spatial (as noted in Mellibovsky et al. 2009) and a temporal sense with increasing $R e$, culminating, if the dynamics are suitably restricted as well, in what appears to be a simple spatially localized TW. Section 4 then 


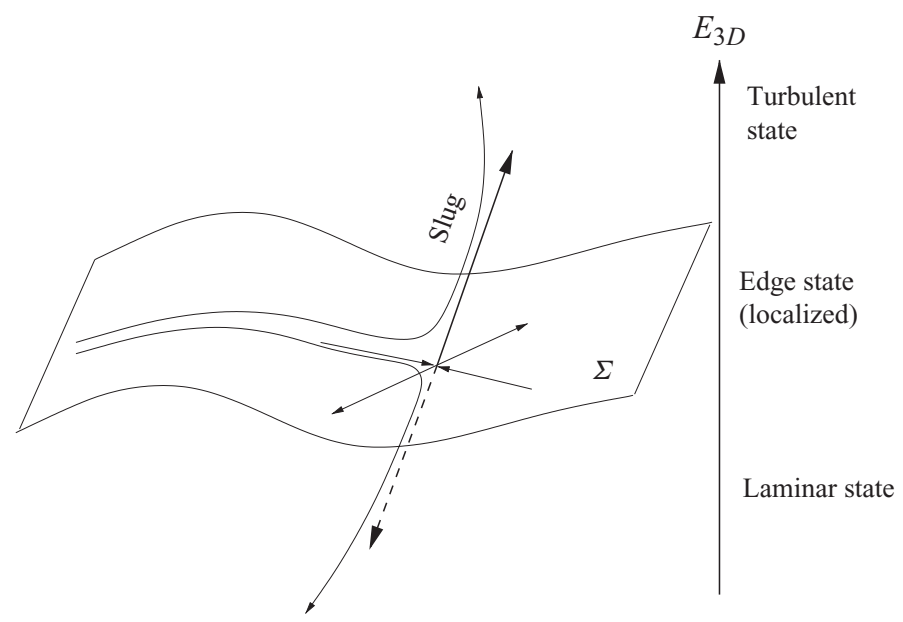

FiguRE 1. Schematic view of the neighbourhood of an unstable exact state (e.g. TW) on the laminar-turbulent boundary $\Sigma$. For $R e>2700$, the part of the unstable manifold which is not tangent to $\Sigma$ and points towards the turbulent state is expected to correspond to a slug in physical space. The symbolic axis $E_{3 D}$ refers to the energy contained in the strictly three-dimensional part of the flow.

studies the generation of a slug when the edge state is perturbed. Particular attention is paid to the shear instability occurring along the edge $\rightarrow$ turbulence route, and to the role of the subsequent vortical fluctuations appearing at the TE in determining whether an equilibrium puff, intermittency or a slug is set up. A discussion follows in $\S 5$.

\section{Numerical procedure}

We consider the incompressible flow of a Newtonian fluid in a straight pipe of cylindrical cross-section and adopt the usual set of cylindrical coordinates $(s, \theta, z)$ aligned with the pipe axis and velocity components, $\boldsymbol{u}=u \hat{\boldsymbol{s}}+v \hat{\boldsymbol{\theta}}+w \hat{\boldsymbol{z}}$. The domain considered here is $(s, \theta, z) \in[0: 1 / 2] \times[0: 2 \pi] \times[0: L]$, where lengths are in units of diameter $D$ and the pipe length is $L$ diameters. The fluid density is chosen to be unity. The flow is described by the incompressible three-dimensional Navier-Stokes equations:

$$
\begin{aligned}
\frac{\partial \boldsymbol{u}}{\partial t}+(\boldsymbol{u} \cdot \nabla) \boldsymbol{u} & =-\nabla p+\frac{1}{R e} \nabla^{2} \boldsymbol{u}, \\
\nabla \cdot \boldsymbol{u} & =0 .
\end{aligned}
$$

where, recall, $R e:=U D / v$, with $v$ being the kinematic viscosity and $U$ the bulk axial velocity. $U$ is held fixed to maintain constant mass flux as in recent experiments (e.g. Peixinho \& Mullin 2006). Energies integrated over the pipe volume are expressed in units of $U^{2} D^{3}$ and time in units of $D / U$. The boundary conditions are periodicity along the pipe length $\boldsymbol{u}(s, \theta, z)=\boldsymbol{u}(s, \theta, z+L)$ and no-slip on the walls $\boldsymbol{u}(1 / 2, \theta, z)=\mathbf{0}$.

\subsection{Definitions}

We use the mixed finite-difference/spectral code described in Willis \& Kerswell (2009) for time-stepping of the incompressible Navier-Stokes equations. Defined on a nonequispaced grid, variables are expanded in the form

$$
\Phi\left(s_{j}, \theta, z, t ; \alpha, m_{0}\right)=\sum_{k=-K}^{K} \sum_{m=-M}^{\boldsymbol{M}} \Phi_{j k m}(t) \exp \left(\mathrm{i}\left(m_{0} m \theta+\alpha k z\right)\right) .
$$


The resolution of a given calculation is described by the triplet $(N, M, K)$, and the set of all complex coefficients defines a phase space of dimension $O(8 M N K)$, which is typically around 2 million. The positive integer $m_{0}$ refers to the discrete rotational symmetry

$$
\boldsymbol{R}_{m_{0}}:(u, v, w, p)(s, \theta, z) \rightarrow(u, v, w, p)\left(s, \theta+\frac{2 \pi}{m_{0}}, z\right),
$$

which can optionally be imposed $\left(m_{0}>1\right)$ or not $\left(m_{0}=1\right)$. The positive real number $\alpha$ is defined by $\alpha=\pi / L$. The coefficients $\Phi_{j k \pm m}$, for instance, can also be linked to impose the mirror symmetry

$$
\boldsymbol{Z}:(u, v, w, p)(s, \theta, z) \rightarrow(u,-v, w, p)(s,-\theta, z) .
$$

Using the notation $\langle\cdot\rangle_{A}$ to denote averaging over the subscripted variable $A$, we define the following energies to describe the perturbations $(u, v, w)$ to the parabolic laminar flow:

$$
\begin{gathered}
E_{\text {roll }}(z, t):=\frac{1}{2} \int_{0}^{1 / 2} \int_{0}^{2 \pi}\left(\left(u-\langle u\rangle_{\theta}\right)^{2}+\left(v-\langle v\rangle_{\theta}\right)^{2}\right) \mathrm{d} \theta s \mathrm{~d} s \\
E_{\text {streak }}(z, t):=\frac{1}{2} \int_{0}^{1 / 2} \int_{0}^{2 \pi}\left(w-\langle w\rangle_{\theta}\right)^{2} \mathrm{~d} \theta s \mathrm{~d} s \\
E(t):=\frac{1}{2} \int_{0}^{L} \int_{0}^{1 / 2} \int_{0}^{2 \pi}\left(u^{2}+v^{2}+w^{2}\right) \mathrm{d} \theta s \mathrm{~d} s \mathrm{~d} z \\
E_{2 d}(t):=\frac{1}{2} \int_{0}^{1 / 2} \int_{0}^{2 \pi}\left(\langle u\rangle_{z}^{2}+\langle v\rangle_{z}^{2}+\langle w\rangle_{z}^{2}\right) \mathrm{d} \theta s \mathrm{~d} s \\
E_{3 d}(t):=\frac{1}{2} \int_{0}^{L} \int_{0}^{1 / 2} \int_{0}^{2 \pi}\left(\left(u-\langle u\rangle_{z}\right)^{2}+\left(v-\langle v\rangle_{z}\right)^{2}+\left(w-\langle w\rangle_{z}\right)^{2}\right) \mathrm{d} \theta s \mathrm{~d} s \mathrm{~d} z
\end{gathered}
$$

$E_{\text {roll }}(z, t)$ represents the kinetic energy associated with the non-axisymmetric crossstream flow and $E_{\text {streak }}(z, t)$ is the kinetic energy associated with the non-axisymmetric axial component of the flow at a given cross-section of the pipe. $E$ is the total kinetic energy associated with the perturbation, $E_{2 d}$ is the two-dimensional counterpart associated with the streamwise-averaged flow and $E_{3 d}$ is the part of $E$ associated with streamwise-dependent modes.

\subsection{Tracking the laminar-turbulent boundary: bisection}

In order to constrain a phase-space trajectory to stay on the laminar-turbulent boundary $\Sigma$, we use the shooting method introduced by Toh \& Itano (1999) and more recently refined by Schneider et al. (2007b). A long turbulent trajectory is simulated and a snapshot $\boldsymbol{u}^{*}$ of relatively low three-dimensional disturbance energy $E_{3 d}(t)$ is selected. An initial condition is then constructed using this field,

$$
\boldsymbol{u}_{\beta}:=\left\langle\boldsymbol{u}^{*}\right\rangle_{\theta}+\beta\left(\boldsymbol{u}^{*}-\left\langle\boldsymbol{u}^{*}\right\rangle_{\theta}\right)
$$

parametrized by a real positive number $\beta$. (The method also works with axial averaging.) The initial condition $\boldsymbol{u}_{\beta=0}$ is axisymmetric and hence cannot trigger turbulence, whereas $\boldsymbol{u}_{\beta=1}$ is the original turbulent state (or more generally an initial state which leads to turbulence). This interval, $\beta \in[0,1]$, is bisected until its width is close to that of machine precision, such that, at the upper limit, the initial condition develops into turbulence, and, at the lower limit, it relaminarizes. This forces the 


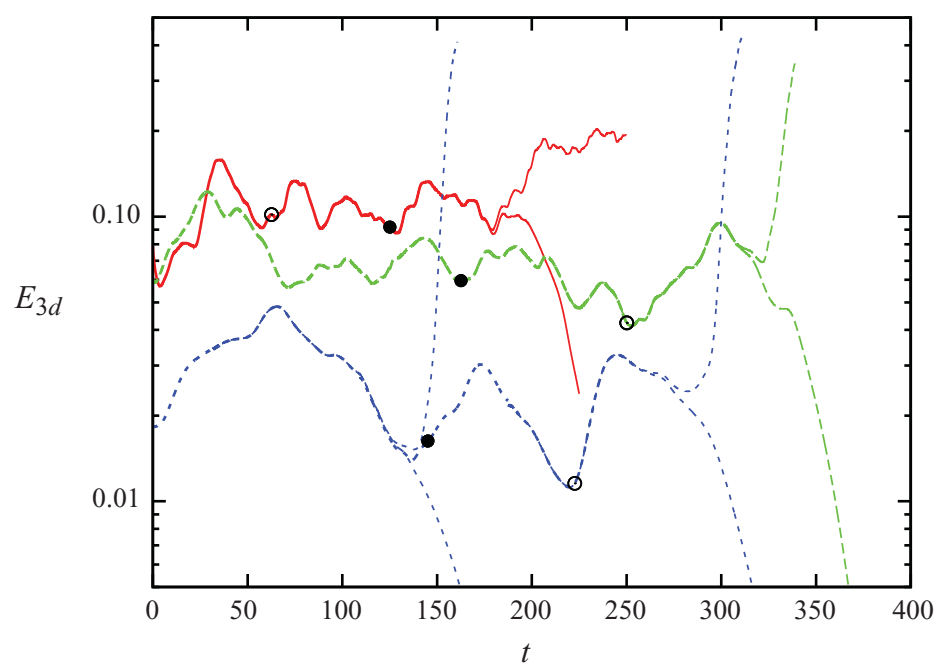

Figure 2. (Colour online) $E_{3 d}(t)$ of the edge trajectories for $R e=2000$ (red solid lines), 3000 (green long-dashed lines) and 6000 (blue dashed lines). The filled circles indicate the edge states shown in figure 3 (time is in units of $D / U$ ). Filled (respectively open) circles also correspond to the cross-sections in figure $6(a, c, e)$ left (respectively $b, d, f)$.

trajectory to stay close to the laminar-turbulent boundary for typical times of $O(300 \mathrm{D} / U)$. For the values of $R e$ used here, the values of $E_{3 d}(t)$ on $\Sigma$ are one or several orders of magnitude below those of the turbulence state, thus making the determination of $\beta$ unambiguous. After bisection, this process can be restarted from nearby states saved near the end of the edge trajectory.

\section{Flow on the laminar-turbulent boundary}

\subsection{Edge states for $R e=2000,3000$ and 6000}

Calculations are now presented for edge trajectories in a periodic domain of length $L=33.51 D$ corresponding to $\alpha=\pi / L=3 / 32$ at $\operatorname{Re}=2000,3000$ and 6000 . A numerical resolution of $(N, M, K)=(40,24,256)$ for $\operatorname{Re} \lesssim 4500$ and $(60,48,384)$ for $4500 \lesssim R e \leqslant 6000$ proves sufficient to ensure that the power spectrum drops by at least seven decades from lowest to highest order modes for both the edge regime and the turbulent regime. The computational domain is also long enough to simulate localized turbulence (puffs) at $R e=2000$ and expanding slugs for $R e \geqslant 3000$. Figure 2 shows typical time traces of $E_{3 d}(t)$ during the application of the bisection algorithm, for initial conditions consisting of various turbulent puff snapshots calculated at $R e=2000$. Two features are immediately clear as $R e$ increases: the traces display smoother (lower-frequency) oscillations with more prominent dips, and the mean value of $E_{3 d}$ decreases. These observations are consistent with previous work in short pipes (see figure 5 of Schneider \& Eckhardt 2009 and figure 7 of Willis \& Kerswell 2009), where it is also clear that the edge energetically approaches the laminar state and diverges away from the turbulent state when $R e$ increases (see figures 5 and 7 of Schneider \& Eckhardt 2009). The situation is not so simple in a longer domain, as figure 3 indicates. Here snapshots of the axial vorticity are plotted after the edge has been tracked for $O(150 \mathrm{D} / U)$, showing that the flow stays localized in the axial direction for all three $R e$. Localization is to be expected for $R e=2000$, where the turbulent state is also localized, but is relatively surprising for $R e \gtrsim 2300$, where 

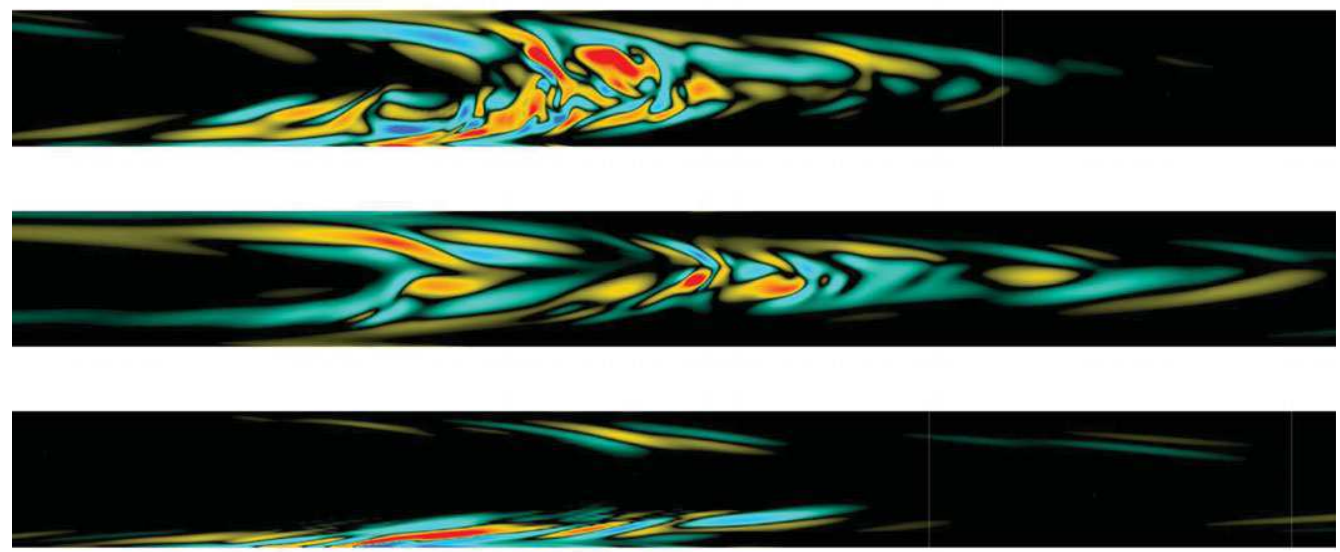

FIGURE 3. (Colour online) $(s, z)$ cut of the axial vorticity field at snapshots on the edge trajectories shown in figure 2 (filled circles). From top to bottom, $R e=2000(t=125 D / U)$, $R e=3000(t=162.5 \mathrm{D} / U), \operatorname{Re}=6000(t=145 \mathrm{D} / U)$. The axial dimension has been scaled by a factor of 2 and only $25 D$ of the $33.51 D$ computational domain is shown.

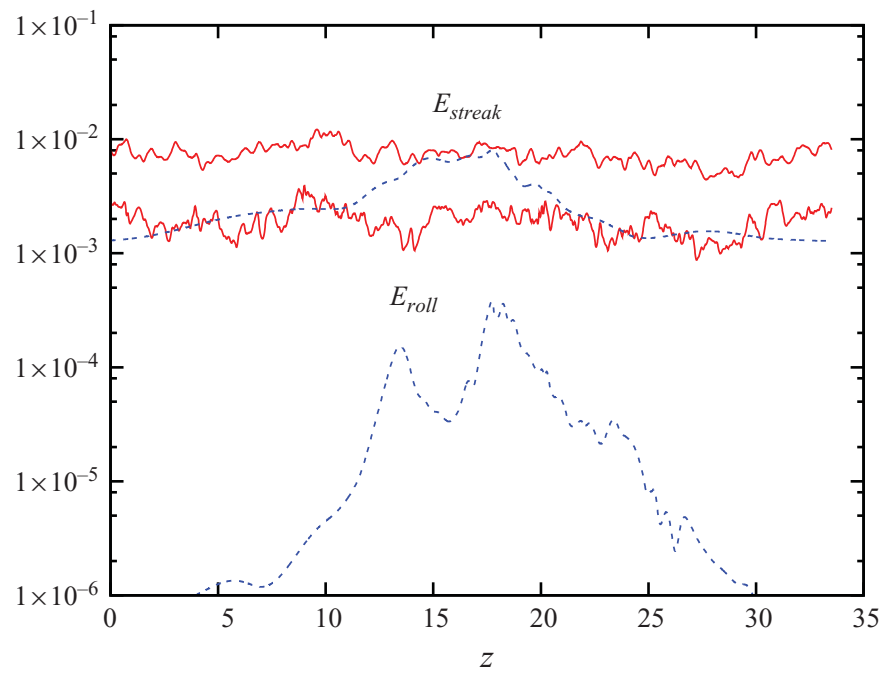

Figure 4. (Colour online) The result of an edge calculation at $R e=3000$ to show localization starting from a globally turbulent initial condition. $E_{\text {roll }}(z, t)$ and $E_{\text {streak }}(z, t)$ plotted at $t=0$ (red solid lines) and $t=84 \mathrm{D} / U$ (blue dashed lines).

turbulence expands spatially once triggered. This edge state localization has already been noted in a $(2+\epsilon)$-dimensional model of pipe flow (Willis \& Kerswell 2009) and, for $R e \leqslant 2800$, in fully resolved computations (Mellibovsky et al. 2009). It was observed in a plane Couette flow (Duguet, Schlatter \& Henningson 2009; Schneider, Marinc \& Eckhardt 2010) as well. Figure 4 confirms that a localized edge state is still reached, even if the starting state for the bisection process is a delocalized turbulent velocity field computed at $R e=3000$. Figure 4 also shows that the local level of $E_{\text {streak }}(z)$ of the edge state in the most energetic region has the same order of magnitude as for the turbulent state, whereas $E_{\text {roll }}(z)$ is everywhere at least one decade lower. The plots in figure 3 indicate that the vorticity fluctuations are more 


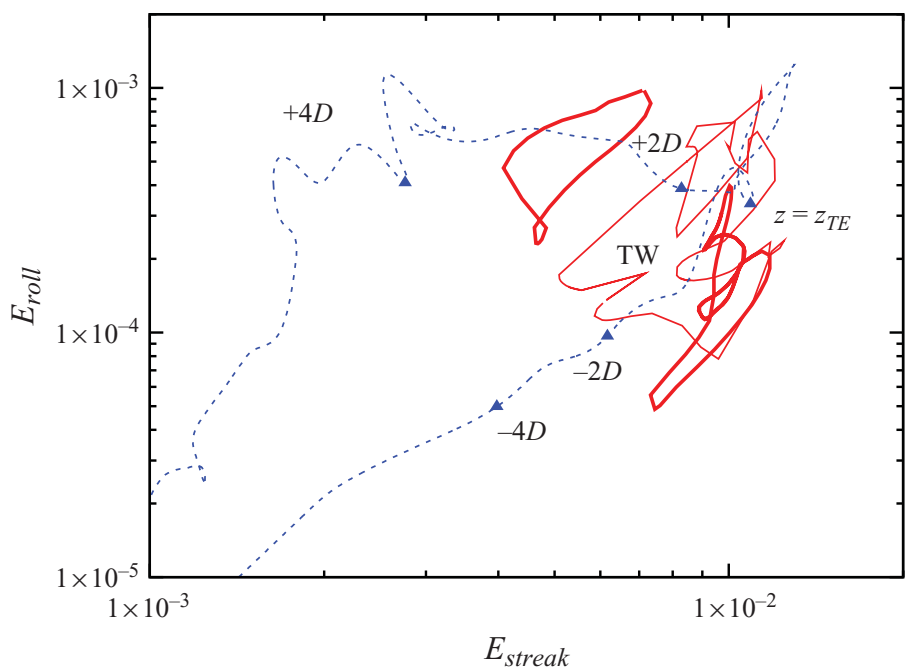

FiguRE 5. (Colour online) Instantaneous phase-space projection on a $\left(E_{\text {streak }}(z), E_{\text {roll }}(z)\right)$-plane for $R e=2000$. Short-domain travelling wave solutions (parametrized by their wavelength $\alpha$, in red solid lines), edge state (blue dashed lines). The axial coordinate is indicated along the trajectories, along with the position of the trailing edge $z_{T E}$.

and more exclusively located in the vicinity of the walls as $R e$ increases, culminating for $R e=6000$ in a radially and azimuthally localized distribution. This is a persistent feature of other edge trackings, after a short transient in which information from the initial state is lost. The implication is clearly that there is an attracting 'edge state' within the edge manifold, as occurs in a short $5 D$ pipe, but here, in addition, the edge state is axially localized. Its energy $E_{3 d}$ as well as temporal complexity decrease as $R e$ increases. The spatial structure becomes simpler as well in the sense that the edge state is more and more radially localized as $R e$ increases.

The edge state at $R e=2000$ has a sharp TE and a less abrupt LE so that it resembles a turbulent puff. The heart of the edge state, however, looks less vigorous than that of the puff. This is borne out in the associated perturbation energy which is an order of magnitude lower in the edge state than for the puff. A useful diagnostic for the axial structure of a spatially inhomogeneous flow structure is to project onto the two-dimensional space of $\left(E_{\text {streak }}, E_{\text {roll }}\right)$ (for a puff, see figure 5 , Willis \& Kerswell 2008). If the structure is axially localized, far upstream and far downstream, both these quantities go to zero as the flow is laminar. Otherwise, the size of the ratio $E_{\text {rolls }} / E_{\text {streaks }}$ is a good indicator of the underlying dynamics: small values of $\lesssim O(0.05)$ are representative of streak-dominated transitional flows and the host of all exact TW solutions known so far (see, for instance, Pringle et al. 2009), whereas $O(0.3)$ values (e.g. the ratio peaks at 0.6 for $z=z_{T E}$ in figure 5, Willis \& Kerswell 2008) indicate significantly more cross-stream dynamics, as seen in developed pipe turbulence. Willis \& Kerswell (2008) found that the TE region of the puff energetically corresponds to fully developed turbulence, implying the existence of regions upstream and downstream where the flow is transitional. Here the flow associated to the edge state is found to fleetingly resemble the regular arrangement of slow and fast streaks reminiscent of the exact TW solutions. The equivalent plot for the edge state at $R e=2000$ (figure 5) shows that the TE (indicated by $z=z_{T E}$ ) has $E_{\text {rolls }} / E_{\text {streaks }} \approx 0.03$ as opposed to 0.6 for a turbulent puff (Willis \& Kerswell 2008) and hence is firmly 


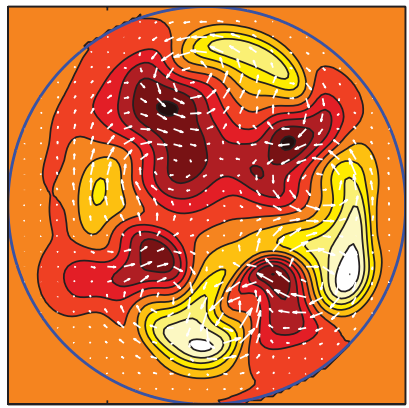




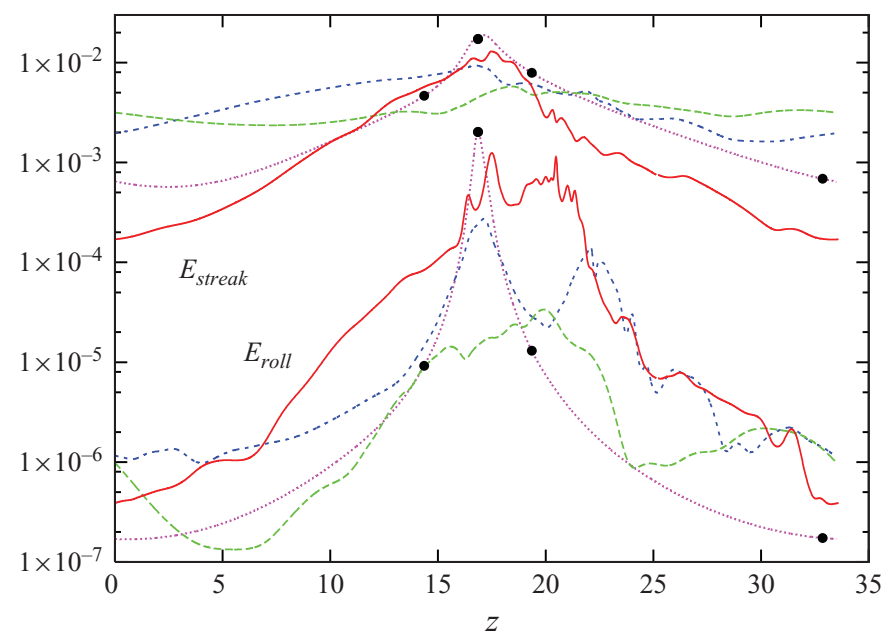

FIGURE 7. (Colour online) $E_{\text {roll }}(z)$ and $E_{\text {streak }}(z)$ energies for the edge state at $R e=2000$ (red solid lines) 3000 (blue dashed lines) and $R e=6000$ (green long-dashed lines). Also shown (magenta dotted lines) are the corresponding quantities (multiplied by 100) for the localized TW found in the $2+\epsilon$ model. Black dots on these curves at 14.3,16.8, 19.3 and 32.8 indicate the locations of the snapshots shown in figure 10.

in figure $6(b)$ displays an arrangement of two streaks. This suggests two TWs with rotational symmetries of $\boldsymbol{R}_{4}$ and $\boldsymbol{R}_{2}$, respectively.

Figure 3 also shows in which sense the spatial structure of the edge state becomes smoother as $R e$ is increased. The strong large-scale fluctuations fill the whole pipe cross-section at $R e=2000$, but the fraction of the cross-section filled by such fluctuations clearly shrinks from $R e=2000$ to $R e=6000$. In the last case (see figure $6 e$ ), the vorticity of the perturbation seems to be concentrated in the radial and azimuthal directions also, with a predominantly laminar flow near the centreline. Cross-sectional velocity fields for $R e \geqslant 3000$ at the TE $\left(z=z_{T E}\right)$ (figure $\left.6 c-f\right)$ again show a large degree of coherency with fast and slow streaks appearing together as large-scale structures. The velocity field at $R e=6000$ is particularly interesting as it looks like a 'minimal coherent state': a pair of streamwise vortices producing a single high-speed streak sandwiched between two low-speed streaks (see figure $6 e, f$ ). This is reminiscent of, but complementary to, a larger-scale structure found in short pipes through edge tracking (Schneider et al. 2007b; Duguet et al. 2008a; Mellibovsky \& Meseguer 2009) and to the TW $S_{1}$ obtained by direct computation (Pringle \& Kerswell 2007; Pringle et al. 2009). The flow at $t \approx 150$ (in figure $6 e$ ) has vortices in the opposite sense compared to $S_{1}$ - a slow streak is sandwiched between two fast streaks - and remains larger-scale (see e.g. figure 3, Viswanath 2009). The flow at $t \approx 220$ (figure $6 f$ ) very closely resembles the TW $S_{1}$. Figure 7 indicates that the peak axial roll energy decreases more sharply than the corresponding streak energy as $R e$ increases. At all three $R e$, the edge states travel in the streamwise direction with a time-dependent velocity which is larger than the bulk velocity $U$.

\subsection{Edge states in reduced symmetry subspaces}

It is natural to ask what form the edge state will ultimately take given that it simplifies in both space and time as $R e$ increases. Taking $R e$ much larger than 6000 proves expensive, with the danger that the bisection algorithm can be misled by numerical instabilities imitating trajectories becoming turbulent. A partial alternative 


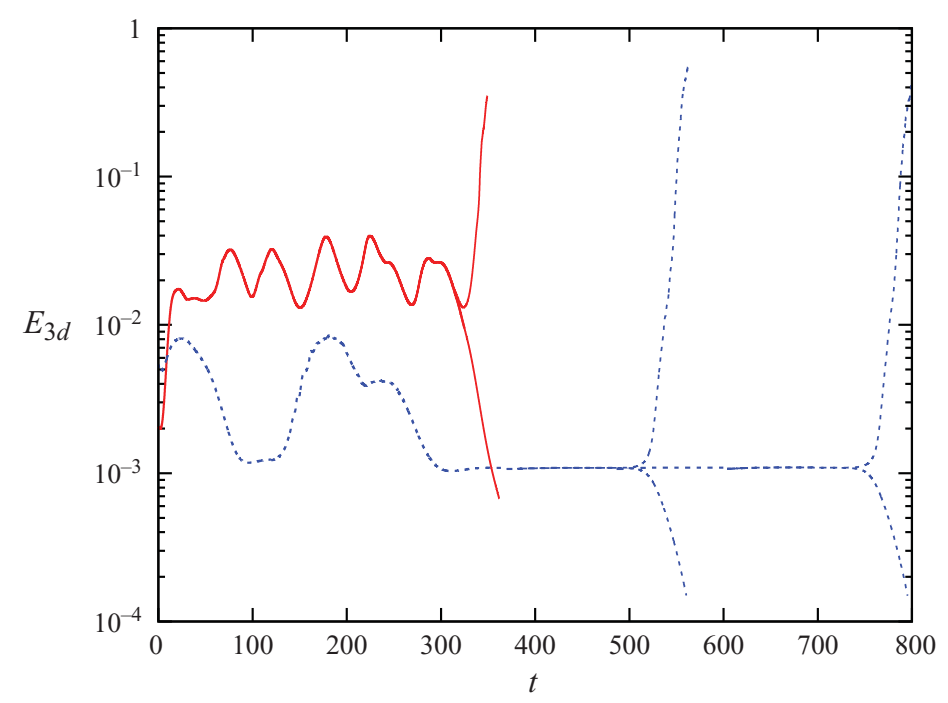

FIGURE 8. (Colour online) $E_{3 d}(t)$ during the bisection process at $R e=8000$ restricted to a $\boldsymbol{R}_{3}$ and $\boldsymbol{Z}$-symmetric subspace using a resolution $(50,8,256)$ (red solid lines) and for the $(2+\epsilon) d$ model $\left(m_{0}=3\right)$ at $R e=10^{4}$ (blue dashed lines).

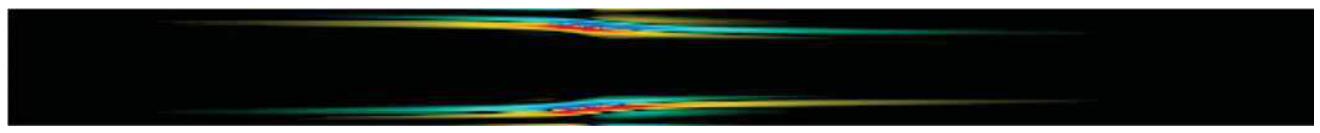

Figure 9. (Colour online) Axial vorticity of the localized edge state in the $(2+\epsilon) d$ model: $R e=10^{4}$ and $11.2 \mathrm{D}$ is shown of the $33.51 \mathrm{D}$ computational domain.

is to restrict the flow dynamics to dynamically consistent subspaces to explore the issue there (Duguet et al. 2008a). Edge states found in these subspaces are embedded within the 'unrestricted' edge but may not be relative attractors with respect to the full-space dynamics. An edge calculation was performed in an $\boldsymbol{R}_{3}$ - and $\boldsymbol{Z}$-symmetric subspace at $R e=8000$, using a resolution $(50,8,256)$ (i.e. the azimuthal wavenumbers included are $0, \pm 3, \pm 6, \pm 9, \ldots, \pm 24)$. It does indeed show evidence for an edge state with a very low number of active temporal frequencies (see figure 8). The temporal oscillations here suggest the possible existence of relative periodic orbits embedded in a relative attractor on $\Sigma$ (as a localized counterpart of the orbit found in Duguet, Pringle \& Kerswell 2008).

A more severe truncation was also adopted, following the ' $2+\epsilon$ '-dimensional model of Willis \& Kerswell (2009) - which allowed many more computations to be done. This model, which is invoked by choosing a truncation $(N, 1, K)$ (and a value for $\left.m_{0}\right)$, is the minimal three-dimensionalization of an axisymmetric simulation, which can qualitatively reproduce the observed flow dynamics in full 3-D (e.g. subcritical transition, localized puffs and expanding slugs). Willis \& Kerswell (2009) revealed a localized edge state for the first time (their figure 9) up to $R e \leqslant 4000$. As in that study, $m_{0}=3$ was imposed, corresponding to retaining only the azimuthal Fourier modes $m=-3,0,3$ in the simulation. Despite this, the resolution in the radial and axial directions still needs to be high $(N=35$ and $K=256)$ to reproduce the radial and axial expansions of the turbulent structures, as well as for accurately capturing the steep interfaces expected in slugs. The trade-off of the modelling is a shift in the 
values of $R e$ characterizing the appearance of these structures. For instance, whereas puff splitting was observed to occur for $R e \geqslant 2300$ in the fully resolved case, this is delayed to 3200 in $(2+\epsilon) d$.

For the $2+\epsilon$ model, the bisection algorithm was run at $R e=10^{4}$, where expanding ('global') turbulence is expected. The initial condition, either localized or delocalized, has no influence on the asymptotic edge results, as mentioned in Willis \& Kerswell (2009). Figure 8 shows the evolution of $E_{3 d}(t)$ during a bisection process. After a transient of duration $300 \mathrm{D} / \mathrm{U}$, the signal $E_{3 d}(t)$ on the edge trajectory converges towards a steady value, which stays constant over very large observation times of $O\left(10^{3}\right) D / U$. This is the signature of convergence towards a relative attractor of constant energy, i.e. a TW solution in the context of a pipe flow. (For analogous plots, see figure 12 in Duguet et al. 2008a for a pipe flow and figure 1 in Schneider et al. 2008 for a plane Couette flow). The phase velocity of the edge state is estimated as $c=(1.06 \pm 0.01) U$, i.e. slightly faster than the bulk velocity. To quantify how well the edge state resembles a TW, the deviation $\delta r(t)$, defined as

$$
\delta r(t)=\lim _{\Delta t \rightarrow 0} \frac{|\boldsymbol{u}(\boldsymbol{x}-c \Delta t \hat{z}, t+\Delta t)-\boldsymbol{u}(\boldsymbol{x}, t)|}{\Delta t|\boldsymbol{u}(\boldsymbol{x}, t)|},
$$

steadily decreased below $10^{-4}$ as the edge trajectory was tracked. Using the Arnoldi code of Kerswell \& Tutty (2007), it was confirmed that this state has only one unstable direction, as expected for an edge attractor.

The spatial structure of the localized edge solution is shown in figure 9, along with the axial distribution of $E_{\text {streak }}$ and $E_{\text {roll }}$ in figure 7 . The axial vorticity is located in a ring-like region of finite radial and axial extents, indicating localization in both senses. Figure 10 shows that the radial concentration is centred on the critical layer at a radius $s \approx 0.69$ consistent with the observed phase speed $c=1.06 U$. At the energy peak, the flow is characterized by a strong azimuthal circulation and a double ring - one inside and one outside the critical layer - of alternating slow and fast streaks. There the streamwise roll pattern undergoes a phase shift of $\pi / m_{0}$ as the critical layer is crossed radially. The presence of the critical layer is less marked $\pm 2.5 D$ upstream and downstream, where only one ring of streaks is present. Further away from the heart of the edge state, the cross-sectional velocities are much smaller and the flow becomes almost streamwise-independent. However, the flow is still dominated by a roll and streak patterns, and cannot be confused with a Hagen-Poiseuille flow. The existence of critical layers within lower branch TW solutions in shear flows was mentioned in Wang et al. (2007) in the context of a plane Couette flow, and by Viswanath (2009) in the context of a pipe flow. It seems to be a generic property of lower branch solutions when $R e \rightarrow \infty$. The streamwise localization is, however, a striking new discovery. Efforts to understand its structure and find similar localized TWs in a three-dimensional pipe flow are currently underway.

\subsection{Summary}

For all values of $R e$ between 2000 and 6000, the spatial structure of the edge state is reminiscent of a turbulent puff with a three-dimensional core region dominated by streaks and streamwise rolls, a sharp arrow-shaped TE interface and a more diffuse LE. As $R e$ is increased, the temporal behaviour of the edge state becomes more regular and the energy concentrates in a narrow region towards the wall so that the central radial region becomes nearly laminar. These trends are particularly exaggerated by the edge state at $R e=10^{4}$ in the $(2+\epsilon) d$ model introduced by Willis \& Kerswell (2009). Here the edge state resembles a localized TW which is steady in a Galilean 


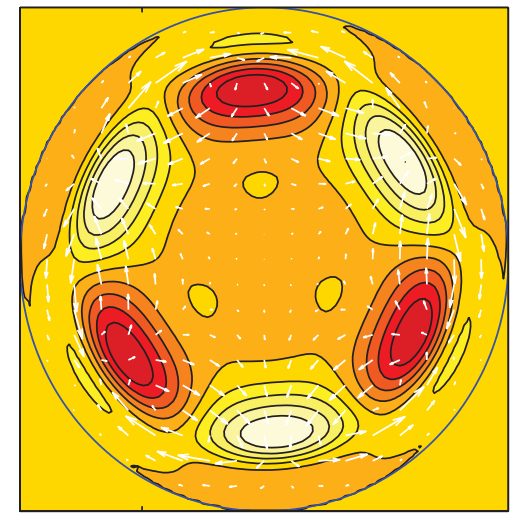




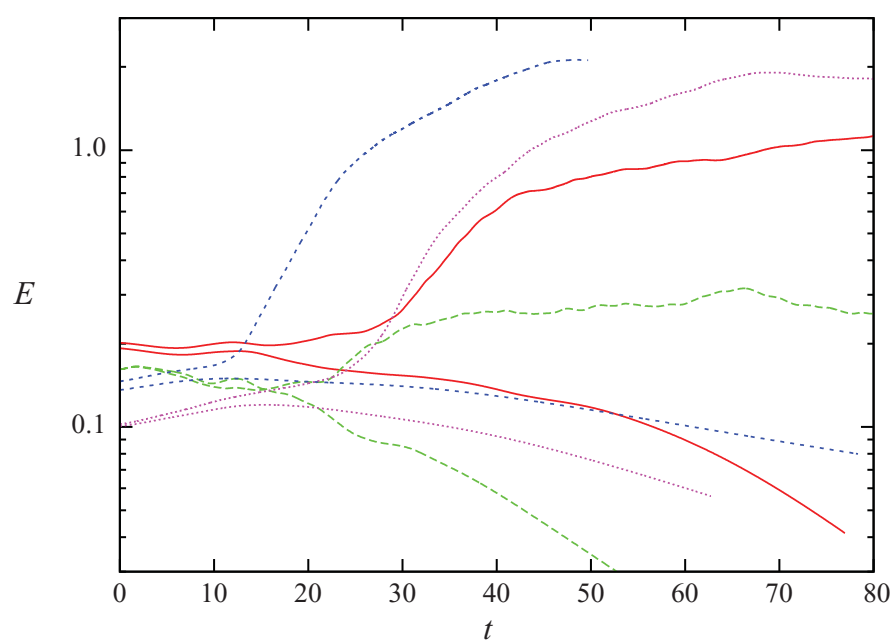

FiguRE 11. (Colour online) Slight perturbations of the perturbation energy $E(t)$, starting from a state on the edge trajectory for a puff, $R e=2000$ (red solid lines) and the expanding slug $R e=3000$ (green long-dashed lines), $R e=4500$ (magenta dotted lines) and $R e=6000$ (blue dashed lines). Upper trajectories lead to turbulence, while the lower trajectories undergo direct relaminarization.

An extension of this thinking to the fully resolved case is relatively straightforward albeit with a couple of nuances. The first is created by the edge state's apparently complicated temporal dynamics, conjectured to be created by a heteroclinic tangle between various TWs (Duguet et al. 2008a, $b$ ). If the edge state has an $n$-dimensional dynamics (e.g. $n=0$ for a steady state and $n=1$ for a periodic orbit), its unstable manifold leading to the turbulent state will be $(n+1)$-dimensional. Trajectories in this manifold are then not formally unique but linked by symmetries of the edge state. It still makes sense to consider any one of these trajectories as defining a characteristic slug evolution for the edge state. The other nuance revolves around whether the edge state is unique or not. Certainly, starting the bisection process off with a variety of different starting conditions leads to what looks to be the same edge state but formally this is difficult to confirm due to its complicated behaviour. Non-uniqueness, however, merely implies that each relative attractor supplies its own route to a globally turbulent state and therefore each has its own corresponding slug evolution. Figure 1 shows a schematic representation of the topology of the neighbourhood of an exact state on $\Sigma$. It is close to figure 14 in Duguet et al. $(2008 a, b)$, with the notable difference that the edge state corresponds to a localized state, whereas the turbulent state is delocalized. The slug corresponds to an escape of the exact unstable solution (here plotted as a generic saddle point) towards the turbulent state.

\section{Slug initiation}

In this section, initial conditions close to the edge state are used to study the genesis of a puff at $R e=2000$ and of slugs at $R e=3000,4500$ and 6000. Simulations are started with the initial conditions $\boldsymbol{u}(\boldsymbol{x}, 0)=(1+\epsilon) \boldsymbol{u}_{\text {edge }}$, where $\epsilon= \pm 0.01$ and $\boldsymbol{u}_{\text {edge }}$ is a snapshot of the edge state with relatively low $E_{3 d}$ (to prolong the evolution phase). Those states have been described in $\S 3.1$, and plotted in figures 3 and $6(b, d, f)$. In both cases, $\epsilon>0$ or $\epsilon<0$, the perturbation energy stays close to that of the edge trajectory for a time of about $10 \mathrm{D} / U$ and then diverges from it (see figure 11). 

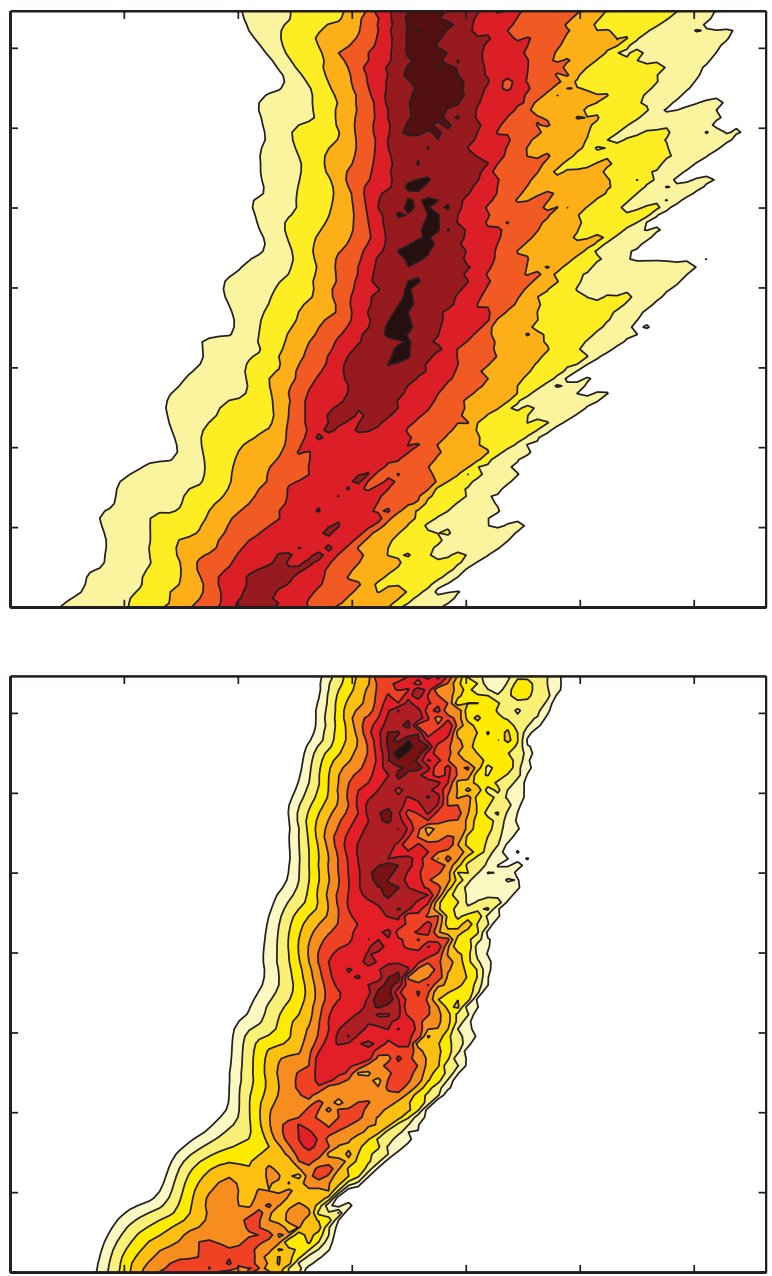
There is a small deceleration of the TE and an even slower deceleration of the LE, so that the flow structure expands slightly. Both processes stop at $t \approx 50$. At this time, the puff looks fully formed and of stable length, with a velocity very close to the bulk velocity $U$. The main feature of the evolution is clearly the intensification of the core region, which is consistent with the findings of Mellibovsky et al. (2009). The edge state is very similar in shape to the turbulent puff, except in the core energy level, so adjustment is small (e.g. the gradients of $E_{\text {roll }}$ are largely constant over time during both the edge and puff stages at either the TE or LE).

Animation of the axial and azimuthal vorticity fields (available online at journals.cambridge.org/flm) in a $(s, z)$-section shows a steady vortex sheet attached near the pipe wall, upstream of the TE. The vorticity gradient across this vortex sheet is clearly observed to increase in magnitude during the edge $\rightarrow$ puff transition. Vortices constantly roll up on top of the vortex sheet, forming wavepackets propagating downstream faster than the turbulent puff (Shimizu \& Kida 2009). These vortical structures had already been pointed out in experiments by Bandyopadhyay (1986). They are qualitatively and quantitatively very reminiscent of the early stage of the Kelvin-Helmholtz (KH) instability for a two-dimensional vortex sheet.

\subsection{Slug genesis at $R e=3000$}

At $R e=3000$, once away from the edge state, the evolution differs strongly from the $R e=2000$ case. Figure 13 shows that the flow initially $(t \approx 5)$ starts to change with a core intensification of $E_{\text {roll }}$ reminiscent of puff formation. This is followed by a sudden deceleration of the TE $(t \gtrsim 10)$, as indicated by $E_{\text {roll }}$, a feature which is absent in the $R e=2000$ case. The main evolutionary stage is the interval $20<t<40$, when the core region immediately downstream of the TE sees steadily increasing gradients in both roll and streak energies. This leads by $t \approx 35$ to high energies downstream and in the centre of the evolving structure. At $t=40$, the roll energy has stabilized at a high level at the centre of the slug while its axial gradients are slowly weakening at the LE. For $t>40$, local maxima of $E_{\text {roll }}$ and $E_{\text {streak }}$ are both to be found near the TE. Downstream of the TE, the average level of $E_{\text {streak }}$ is comparable to the level in the active region of the edge state, while $E_{\text {roll }}$ is everywhere larger than its edge counterpart.

During the escape from the edge state, the propagation speed of the TE, $c_{T E}$, drops from the initial value of $1.40 U$ (the speed of the edge state) to $0.84 U$. In contrast, the speed of the LE, $c_{L E}$, decreases slowly from $1.40 U$ to approximatively $1.18 U$ by the end of the simulation. Experimental measurements by Lindgren (1969) at $R e=3000$ suggest that $c_{L E}=1.13 U$ and $c_{T E}=0.83 U$. This is confirmed by later experiments by Wygnansky \& Champagne (1973), Hof et al. (2005) and Nishi et al. (2008), and compares favourably with the present numerical measurements. Note that even after $t=40$, the iso-contours of $E_{\text {roll }}$ near the LE do not form a straight line in the $(z-U t, t)$ diagram of figure 13. This suggests that the slug expansion at $R e=3000$ is not strictly continuous but occurs by jumps, as pointed out in the experiments by Lindgren (1969) 'just above the threshold for transition'.

The axial vorticity is shown in a series of snapshots across the period $t=10-40$ in figure 14. As for $R e=2000$, vortical structures located on the vortex sheet behind the TE start to grow in amplitude at $t=25$ on top of the vortex sheet. In their initial stage, these vortical structures are again very reminiscent of the rolling-up vortex sheet observed in the early stage of the $\mathrm{KH}$ instability. The presence of roll-up fluctuations is also evident in figure 15 at $R e=4500$. While, for $R e=2000$, these fluctuations catch up with the active region, here some of the vortices stay initially 


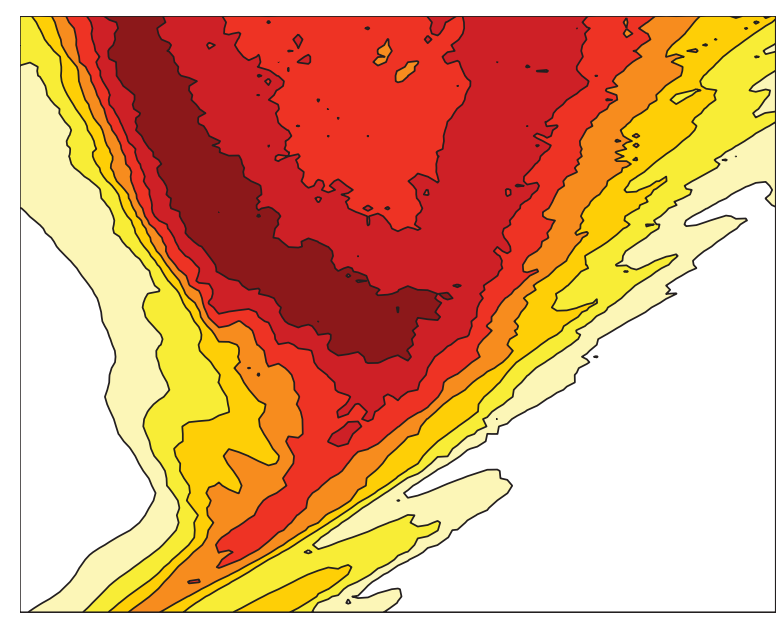


हु?

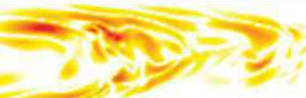
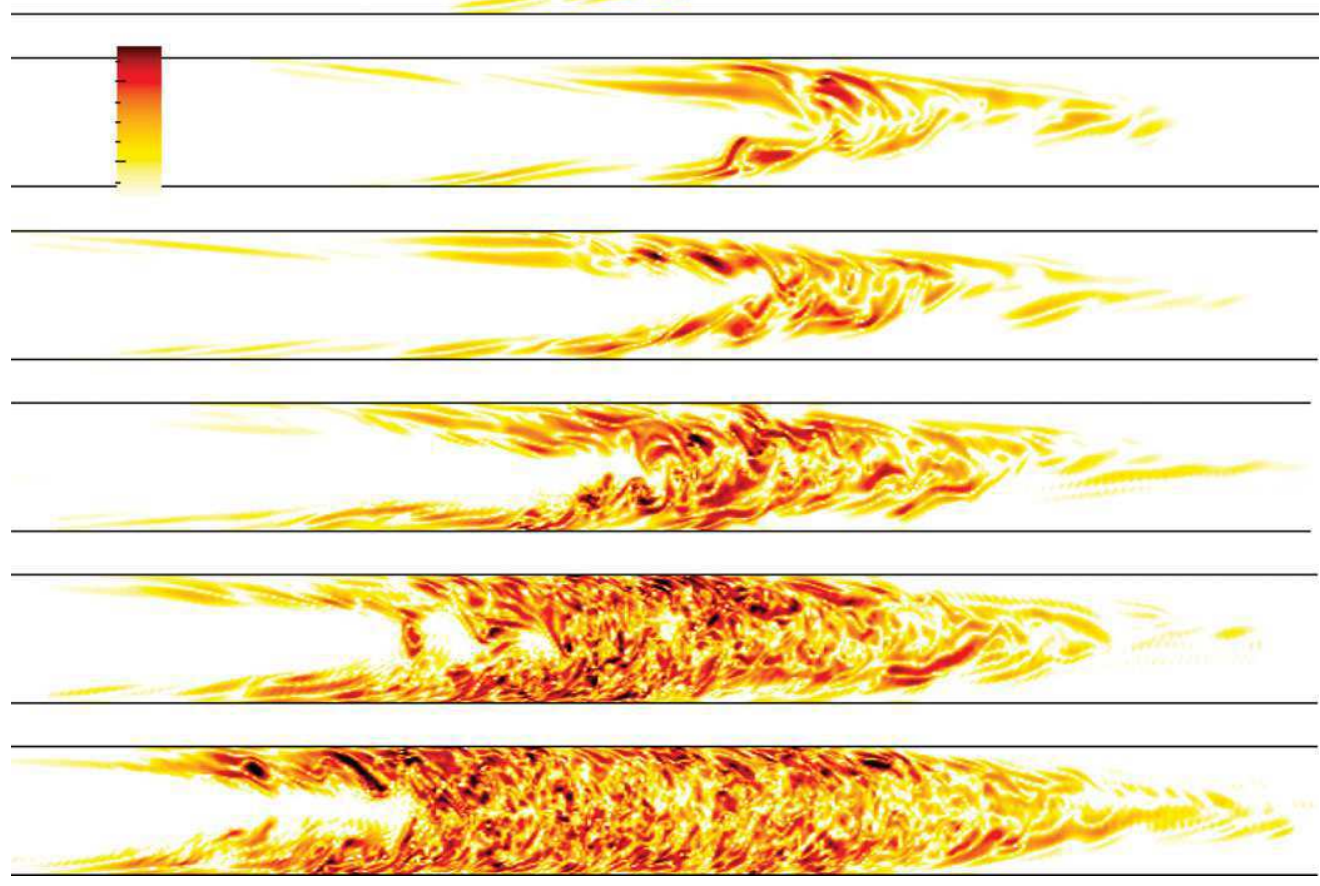

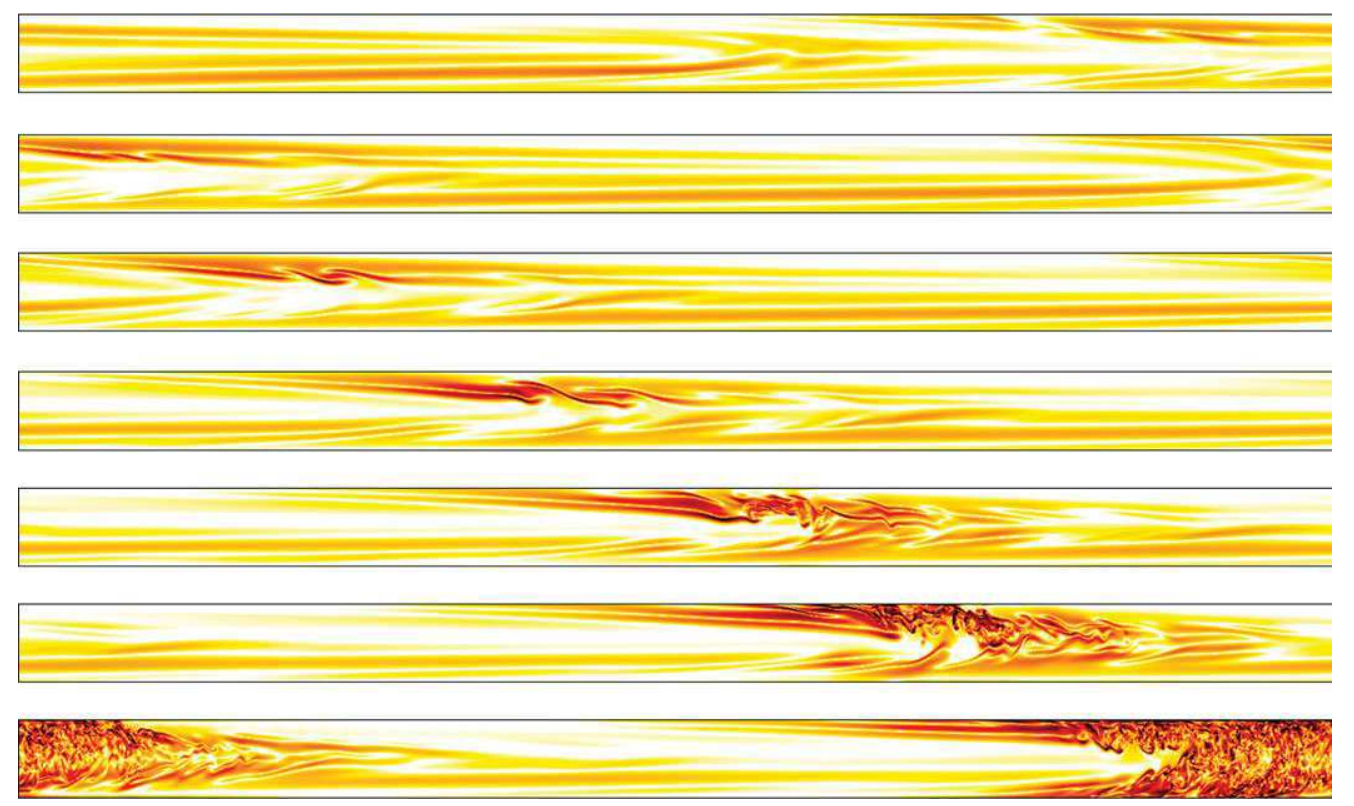

Figure 15. (Colour online) Azimuthal vorticity focusing on the instability of the edge state leading to the early formation of a slug, as shown in figure $16, R e=4500$ (top to bottom, $t=5,12.5,15.75,20.3,25,27.5,35.25 \mathrm{D} / \mathrm{U}$ ) (same scale as in figure 14). Animation available online.

from figure 16 yields $c_{T E}=1.47 U$ and $c_{L E}=0.68 U$ at $R e=4500$. This is in very good agreement with experimental estimates, given the difficulty in measuring the speed of the LE experimentally. However, the most striking feature of the $E_{\text {roll }}$ and $E_{\text {streak }}$ evolution plots is now the large gradient of $E_{\text {roll }}$ at the LE. This is consistent with experimental observations, but is nevertheless eye-catching compared to the more diffuse LE for $R e=2000$ and 3000 .

A typical sequence of plots visually representative of the elongation mechanism of the slug is shown in figure 18 for $R e=4500$. The quantity displayed is the azimuthal vorticity of the perturbation to the base flow. As for $R e=3000$, intense vortical structures start to develop near the vortex sheet behind the TE. Again, these vortical structures clearly resemble a rolling-up vortex sheet observed in the early stage of the KH instability. Their propagation velocity is initially slower than the overall speed of the turbulent region from which it originates, thus inducing a time delay: the vortical fluctuations are left behind the passing slug. The destabilization of these vortical fluctuations results in an ejection process, leading to the formation a local blob of turbulence. Initially this blob is clearly distinct from the turbulence in the active region already developed at earlier times; however, its vortical intensity becomes quickly comparable to the levels observed at the TE. This localized blob of turbulence migrates towards the centreline, invading the whole cross-section of the pipe. Immediately after it aggregates to the existing slug. The global elongation of the turbulent structure apparently results from an intermittent sequence of such repeated ejection $\rightarrow$ destabilization $\rightarrow$ aggregation events (see video online).

For $R e=6000$, the azimuthal vorticity of the perturbation to the base flow is shown in figure 19. Just like the evolution at $R e=3000$ and 4500, the flow departs from the edge state through a clear roll-up of the vortex sheet at $t \approx 12$. However, the 


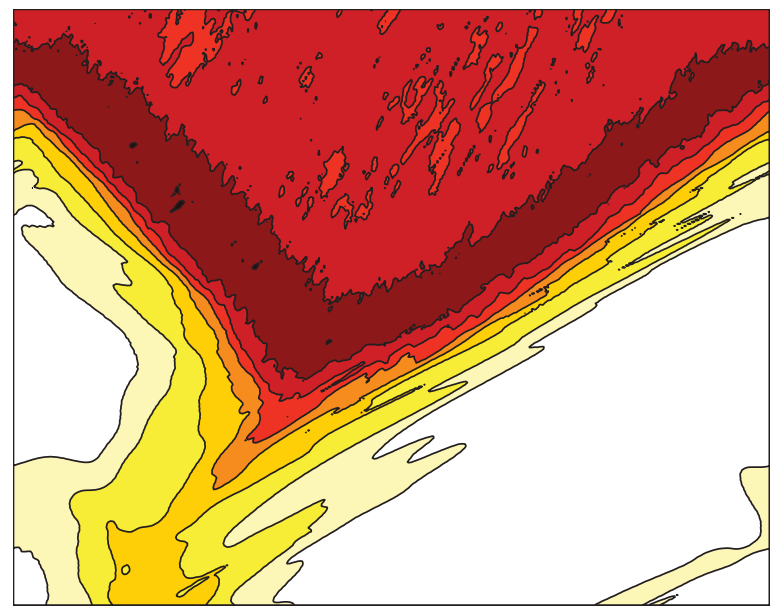




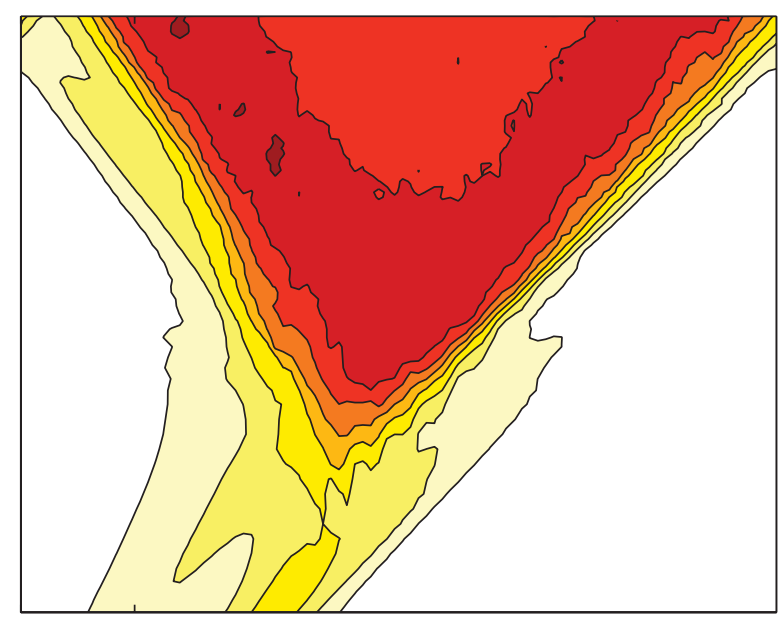




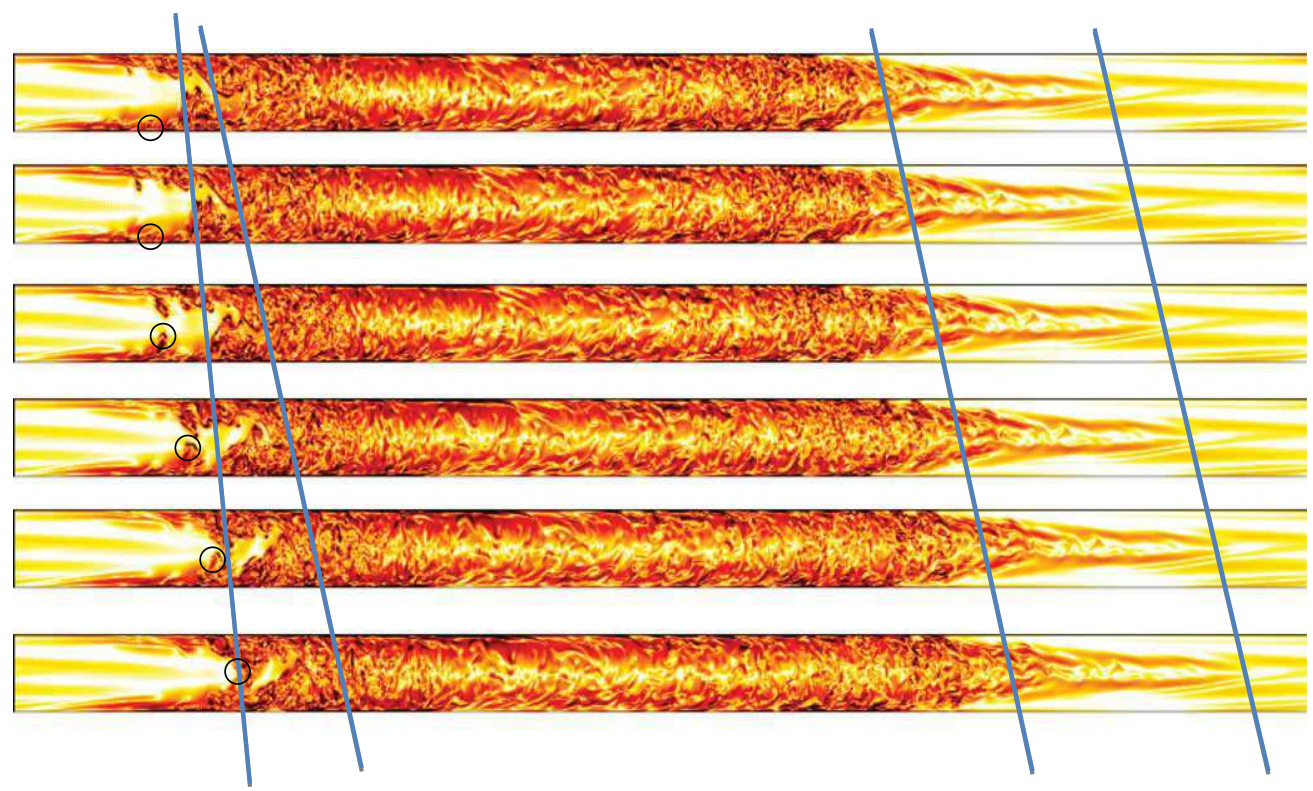


from the large gradients across the vortex sheet, plays a key role in the sustenance mechanism of the turbulent puff: these vortices propagate downstream with a velocity greater than that of the puff; hence, they represent the missing link to feed turbulent fluctuations back into the turbulent puff. The loop is plausibly closed because the $\mathrm{KH}$ fluctuations travel at a phase velocity faster than that of the puff itself.

We now wish to investigate how this mechanism is altered when turbulent puffs are no longer equilibrium states. Vortical structures growing on top of the vortex sheet near the TE are also detected by eye in figures 14, 18 and 19. Animation of the case $R e=6000$ (expanding slug) clearly shows that all fluctuations ejected from the TE interface travel slower than the vortex sheet from which they originate. In contrast, at $R e=2226$ in Shimizu's data (localized puff), the KH fluctuations are faster than the puff at least in the radial region between the pipe axis and the vortex sheet. Given this, we focus here on the axial propagation speed of the vortical structures and compare it with the corresponding speed of the turbulence as $R e$ varies. The main idea here is that the value of $c_{T E}$ (to be compared eventually to $c_{L E}$ ) is controlled solely by the vortex dynamics at the TE, which in turns depends on both the initial vortex propagation speed $C_{K H}$ and a representative speed of the turbulence. Since in a developed slug both interfaces travel at different velocities, the question arises which quantity should be taken as a reference velocity of the bulk turbulence. From figures 13,16 and 17 as well as from the animations online, it is clear that the sudden deceleration of the TE is responsible for the elongation, and that the instability mechanisms acting during the edge $\rightarrow$ slug evolution act at the upstream interface. On the contrary, the LE behaves as a passive region connecting the turbulent zone to the laminar downstream of it. It is characterized by a front propagation speed $c_{L E}$, which appears to be dependent only on $R e$, independently of whether there is a upstream interface and of the possible dynamics occurring there. For instance, in a high-Re experimental pipe set-up where disturbances would be injected continuously near the inlet, a propagating front delimiting the turbulence and the laminar flow would plausibly travel downstream at the same velocity $c_{L E}$, whereas in that case there is no such thing as a TE. It thus appears natural, at a given value of $R e$, to regard $c_{L E}$ as the propagation speed of the turbulence through a laminar environment. Note that understanding the experimentally observed dependence of $c_{L E}$ on $R e$ is an open issue, whose determination undoubtedly necessitates full knowledge of the distribution of the Reynolds stresses inside the turbulent zone. Rationalizing this is beyond the scope of the present investigation. Instead, $c_{L E}$ as an observed function of $R e$ is adopted here as a representative of the speed of the turbulence as a whole.

The method used to track each individual $\mathrm{KH}$ vortex is relatively simple: the location of localized maxima of the azimuthal vorticity perturbation $\omega_{\theta}$ is tracked against time in a given $(s, z)$-section. The axial velocity $C_{K H}$ of each ejected vortex is then interpolated numerically by a linear fit. This method relies on an unambiguous identification of intense peaks of $\omega_{\theta}$ in the chosen plane. Such a condition is not fulfilled in practice for all vortex events, for instance, if the chosen vortex drifts away from the chosen $(s, z)$-plane, or if the location of the maximum is too patchy to be accurately tracked. The number of in-plane vortices which can be accurately tracked in our videos (available online) is $O(15)$, which appears just sufficient to get useful statistics. The axial velocity of all tracked vortices is shown in figure 20 against their radial position for $R e=2000,3000,4500$ and 6000 . The spread in the data necessarily calls for a statistical description of the process. All perturbations have their maximum located between $s=0$ and $s=0.28$ (in units of $D$ ). Their instantaneous axial velocity lies between $U$ and $2 U$ (respectively the puff velocity and the centreline velocity 


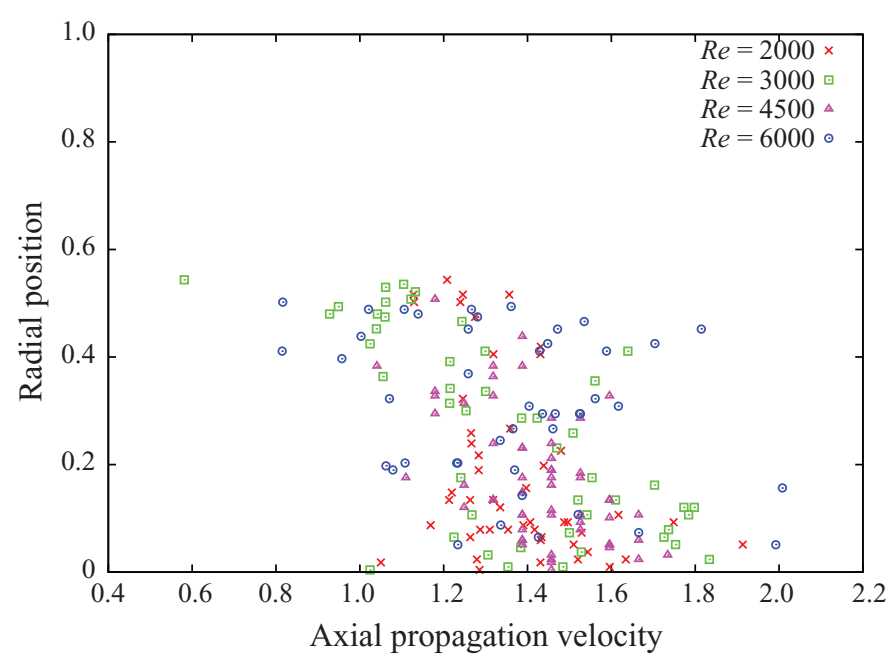

Figure 20. (Colour online) Axial velocity of the KH vortices as a function of their radial position (here in units of the radius $D / 2$ ), for $R e=2000,3000,4500$ and 6000 .

of the laminar flow) at $R e=2000$. For $R e \geqslant 3000$, it lies between $0.6 U$ and $2 U$. Importantly, the distribution of $C_{K H}$ resembles neither the laminar parabolic profile nor the turbulent one, which suggests that we are not trivially measuring the passive advection of perturbations by the flow.

The axial velocity $C_{K H}$ in the database was averaged over the whole vortex population for each value of $R e$. The mean value $\left\langle C_{K H}\right\rangle$ varies with $R e$ between 1.34 and 1.38, which essentially suggests that $\left\langle C_{K H}\right\rangle$ is independent of $R e$, as should be expected for the suspected underlying inviscid $\mathrm{KH}$ mechanism. For comparison, we have also used the data in figure 14 by Shimizu \& Kida (2009), which corresponds in our units to a flux-based Reynolds number of $R e=2226$ in a smaller pipe of length $L=25.13 D$. Those authors track the velocity $U_{f}(s, z)$ of perturbations by measuring space-time correlations, and compare it to the puff velocity. The perturbations they also identify with $\mathrm{KH}$ structures are located in the central region $|s| \leqslant 0.3$, for which the $z$-averaged quantity $\left\langle U_{f}\right\rangle_{z}(r)$ varies between $1.15 U$ and $1.35 U$. Despite a different tracking algorithm, we consider this correspondence reassuring, especially given the difference in pipe lengths and the possible influence of a shorter pipe on the puff dynamics.

We now compare the propagation velocity of the $\mathrm{KH}$ vortices to the velocity $c_{L E}$ and $c_{T E}$ of the LE and the TE (respectively), deduced by interpolation from figures 12, 13, 16 and 17 . Our values of $c_{L E}$ and $c_{T E}$ are shown in figure 21 as a function of $R e$. They compare very well with available experimental data by Lindgren (1969), Wygnanski \& Champagne (1973), Hof et al. (2005) and Nishi et al. (2008). Adding now the data for the average speed $\left\langle C_{K H}\right\rangle$, the average speed plus or minus the standard deviation $\left\langle C_{K H}\right\rangle \pm \delta C_{K H}$, and the minimum value min $C_{K H}$ as functions of $R e$ in figure 21, a clear trend is noticeable which we summarize as follows.

(i) For $R e=2000, c_{L E}=c_{T E} \approx 1.03 U$, much below $\left\langle C_{K H}\right\rangle-\delta C_{K H}=1.26 U$ and below $\min C_{K H}=1.05 U$. This is the case where turbulence stays localized inside a turbulent puff structure. As can also be seen from figure 21, all vortices travel faster than the turbulent puff.

(ii) For $R e=3000, c_{L E} \approx 1.16 U$, below $\left\langle C_{K H}\right\rangle$ but slightly above $\left\langle C_{K H}\right\rangle-\delta C_{K H}$ and clearly above $\min C_{K H}=0.93 U$. (Note that we have omitted the possible rogue data 


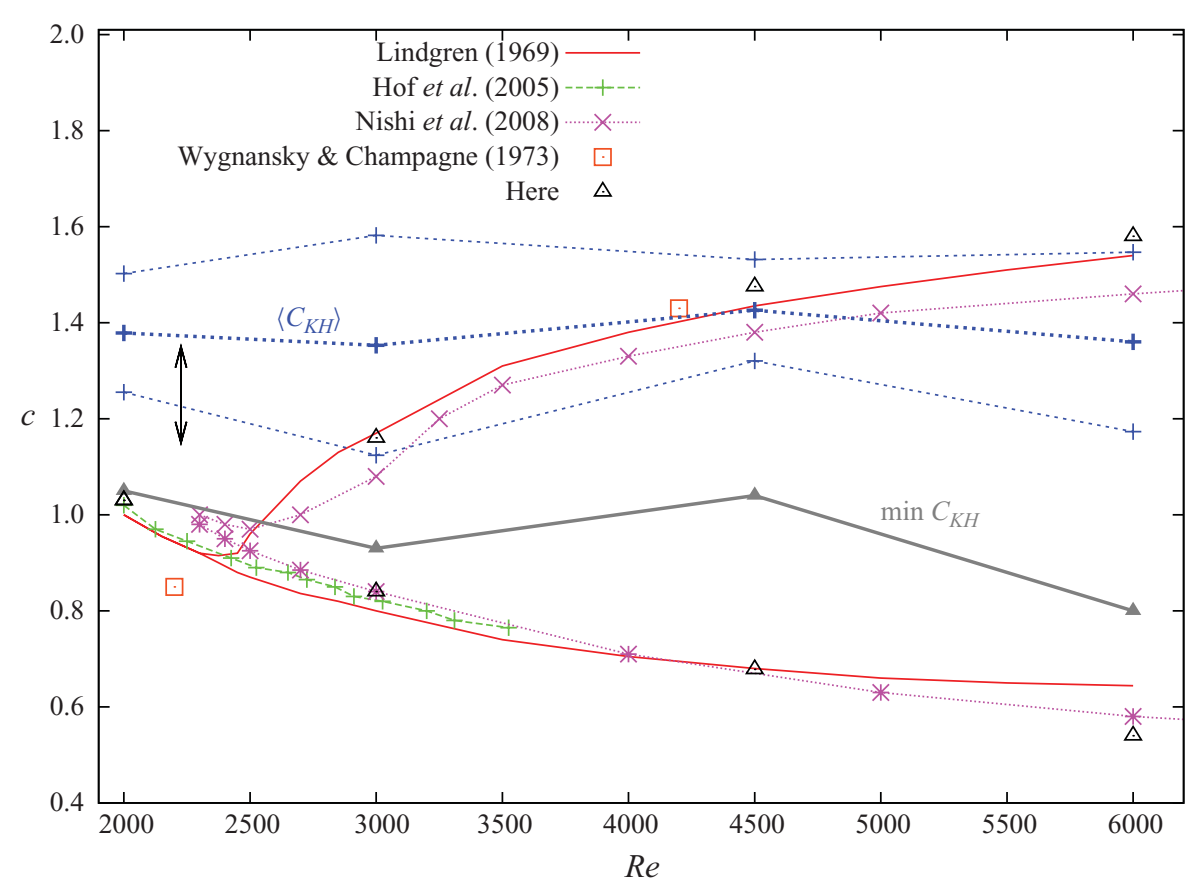

Figure 21. (Colour online) Axial velocity of the LE and the TE as a function of $R e$ (triangles), compared with available experimental data. The velocity of the LE is always greater (slugs) or equal (puffs) to that of the TE. The mean axial propagation velocity $\left\langle C_{K H}\right\rangle$ of $\mathrm{KH}$ vortical structures ejected from the TE is indicated, plus or minus its standard deviation $\delta C_{K H}$ (see $\S 3.4$ ), as well as the minimum value $\min C_{K H}$ among the data at hand. The arrow at $R e=2226$ indicates the statistical velocity of fluctuations computed by Shimizu \& Kida (2009).

point $C_{K H}=0.58 U$ present in figure 20, which would only exaggerate the trend.) This is a slug case where turbulence expands discontinuously through the pipe. According to the statistical description, we note that some of the vortices (the slowest ones) do not catch up with the LE velocity.

(iii) For $R e=4500$ and even more clearly for $R e=6000, c_{L E}$ is above $\left\langle C_{K H}\right\rangle$, close to $\left\langle C_{K H}\right\rangle+\delta C_{K H}$ and much above $\min C_{K H}$. This is a case of continuous slug expansion. Statistically, almost all vortices propagate at a velocity slower than the bulk turbulence.

The interpretation is straightforward: the turbulent state stays localized as long as all $\mathrm{KH}$ vortices travel faster than the LE. Otherwise, it delocalizes, expanding spatially by accretion when at least some of the vortices do not catch up with it. Our analysis for the puff case confirms the observation made by Shimizu \& Kida (2009): all $\mathrm{KH}$ vortices travel faster than the turbulent puff. In the slug case, the sequence of events shown in figure 18 is entirely representative of the dynamics shown in the online videos for $R e=3000,4500$ and $R e=6000$ : slow $\mathrm{KH}$ vortices are ejected towards the centreline, break down in the wake of the turbulent state travelling faster downstream. The original turbulence then elongates through accretion. The process repeats itself until a turbulent flow has invaded the whole pipe. According to figure 21, for larger $R e$, the likelihood of a given $\mathrm{KH}$ vortex catching up with the turbulent structures decreases and the slug expansion can truly be described as continuous 
when (statistically) all vortices contribute through their early breakdown to the axial expansion of the slug.

\section{Discussion}

This paper has examined the likely route through which turbulent slugs are generated by focusing on how the relative attractor (the edge state) on the laminarturbulent boundary breaks down. If the edge state is unique, this can be viewed in some sense as the generic situation, since, for all but the most drastic initial perturbations, the disturbed flow will, at least initially, evolve towards the edge state before being directed away by its unstable manifold. As a result, experiments working with disturbances just above the transitional threshold should see essentially this slug genesis. The flow development along the unstable manifold of the edge state is also a natural way of defining a slug since it is a constantly expanding, evolving state.

For all values of $R e$ examined so far (1800-2800 in Mellibovsky et al. 2009 and up to 6000 here), these edge states are always found to be axially localized despite the fact that the associated turbulent state delocalizes beyond $\approx 2300$. They appear to have chaotic dynamics and look very similar to puffs albeit with less energy especially in their core (Mellibovsky et al. 2009; Willis \& Kerswell 2009). Their internal dynamics are found to smoothen temporally and become even more localized spatially with increasing $R e$, reflecting the fact that the edge state approaches the laminar rather than the turbulent state energetically. Working within symmetric subspaces exaggerates this trend by revealing an almost periodic state and, in $2+\epsilon d$, the simplest state possible, which appears to be a TW.

Once the edge state is weakly perturbed at $R e \geqslant 3000$, slug genesis is observed to occur through three distinct phases:

(i) intensification of the core energy and subsequent front sharpening,

(ii) shear instability of the wall-attached vortex layer set-up at the TE,

(iii) spatial expansion through the contamination of the fluid left behind the TE.

The first two phases are also seen during puff genesis at $R e=2000$. In particular, at all $R e$ studied, the instability of the edge state manifests itself initially by a local increase of energy and sharper velocity gradients at the TE which form an intense vortex sheet near the wall at the TE. This shear is unstable to what looks to be a $\mathrm{KH}$ instability and rolled-up vortices are shed (see, for instance, figure 15). These travel downstream at a $R e$-independent average speed of $\approx 1.4 U$. When $R e$ is low enough, for instance from figure 21 , for approximatively $R e \leqslant 2500$, all vortices travel downstream faster than the developing turbulence, and a puff of stable length forms (Shimizu \& Kida 2009). The turbulence can then be considered in statistical equilibrium. For much larger $R e$, for instance, $R e=6000$ in our simulations, almost all vortices ejected from the vortex sheet are slower than the turbulence yet are intense enough to break down themselves. Precisely because they are slower, these new patches of turbulence are sown behind the passing interface, and end up aggregating to the existing turbulence, creating the impression of an overall smooth elongation. The slug can then be seen as a turbulent puff 'out of equilibrium'. However, the mechanisms through which the vortical activity is sustained within the slug itself are still unclear.

For intermediate values of $R e$, the propagation of the $\mathrm{KH}$ shed vortices has to be investigated statistically; some of them travel faster than the turbulent puff, while the slowest ones cause its spatial elongation. The turbulent puff is still out of equilibrium but its spatial elongation occurs now in jumps, as can be seen, for instance, in figure 13 at $R e=3000$. More extensive data are needed to answer the question as to whether 
the transition from discontinuous to continuous elongation is abrupt or happens continuously when varying $R e$. A last regime of interest is defined by $\min C_{K H} \approx c_{L E}$. In this particular configuration, the puff is nearly in equilibrium but departs from the equilibrium turbulent puff every time a rare statistical event occurs, under the form of a slower KH shed vortex. The turbulent puff is expected to hesitate intermittently between keeping his puff-like shape or expanding spatially.

The range of $R e$ for this intermediate regime can be determined, to a first approximation, by interpolation of $\min C_{K H} \approx c_{L E}$ from figure 21 , which yields $R e \approx 2500$. A more conservative estimate of $R e \approx 2850$ is given by $\left\langle C_{K H}\right\rangle-\delta C_{K H} \approx c_{L E}$, i.e. when only rare statistical events travel slower than $c_{L E}$. This regime may well correspond to the spatiotemporally intermittent dynamics identified by Moxey \& Barkley (2010) for Re between 2300 and 2600. A proper computational identification of this regime demands much larger domains, for instance $L=150 D$ in Moxey \& Barkley (2010), as well as a more accurate way of getting statistics of $C_{K H}$. This regime bridges the gap in $R e$ noticed by experimentalists between puffs and slugs (see, for instance, figure $2 b$ in Wygnanski \& Champagne 1973). The regime dependence on the relative sizes of $\min C_{K H}$ and $c_{L E}$ can be summarized as follows:

(i) $\min C_{K H}>c_{L E}$ : equilibrium puff;

(ii) $\min C_{K H} \approx c_{L E}$ : intermittent regime;

(iii) $\min C_{K H}<c_{L E}$ : slug expansion.

Finally, it would be interesting to investigate how this approach to spatio-temporal intermittency can be extended to other shear flows, such as the expansion of turbulent spots in a plane Couette flow or in the Blasius boundary layer.

Y.D. would like to thank D. S. Henningson for financial support during the years 2008-2009 and P. Manneville for fruitful discussions. A.P.W. was supported by a Marie-Curie Intra-European Fellowship grant.

Supplementary data are available at journals.cambridge.org/flm.

\section{REFERENCES}

Adrian, R. J. 2007 Hairpin vortex organization in wall turbulence. Phys. Fluids 19, 041301.

BANDYOPADHYAY, P. R. 1986 Aspects of the equilibrium puff in transitional pipe flow. J. Fluid Mech. 163, 439-458.

BinNie, A. M. \& Fowler, J. S. 1947 A study of a double refraction method of the development of turbulence in a long cylindrical tube. Proc. R. Soc. Lond. A 192, 32.

Darbyshire, A. G. \& Mullin, T. 1995 Transition to turbulence in constant-mass-flux pipe flow. J. Fluid Mech. 289, 83.

Duguet, Y., Pringle, C. \& Kerswell, R. R. $2008 b$ Relative periodic orbits in transitional pipe flow. Phys. Fluids 20, 114102.

Duguet, Y., Schlatter, P. \& Henningson, D. S. 2009 Localized edge states in plane Couette flow. Phys. Fluids 21, 111-701.

Duguet, Y., Willis, A. P. \& Kerswell, R. R. 2008 a Transition in pipe flow: the saddle structure on the boundary of turbulence. J. Fluid Mech. 613, 255-274.

Eckhardt, B., Schneider, T. M., Hof, B. \& Westerweel, J. 2007 Turbulence transition in pipe flow. Annu. Rev. Fluid Mech. 39, 447-468.

FAisst, H. \& ECKHARDT, B. 2003 Travelling waves in pipe flow. Phys. Rev. Lett. 91, 224-502.

Gibson, J. F., Halcrow, J. \& Cvitanovic, P. 2008 Visualizing the geometry of state space in plane Couette flow. J. Fluid Mech. 611, 107-130.

Gibson, J. F., Halcrow, J. \& Cvitanovic, P. 2009 Equilibrium and travelling wave solutions of plane Couette flow. J. Fluid Mech. 638, 1-24. 
Hagen, G. H. L. 1839 Über die Bewegung des Wassers in engen zylindrischen Röhren. Poggendorfs Annal. Physik Chemie 16, 423.

Halcrow, J., Gibson, J. F., Cvitanovic, P. \& Viswanath, D. 2009 Heteroclinic connections in plane Couette flow. J. Fluid Mech. 621, 365-376.

Herron, I. H. 1991 Observations on the role of vorticity on the stability of wall bounded flows. Stud. Appl. Math. 85, 269-286.

Hof, B., Delozar, A., Avila, M., Tu, X. \& Schneider, T. M. 2010 Eliminating turbulence in spatially intermittent flows. Science 327, 1491-1494.

Hof, B., van Doorne, C. W. H., Westerweel, J. \& Nieuwstadt, F. T. M. 2005 Turbulence regeneration in pipe flow at moderate Reynolds numbers. Phys. Rev. Lett. 95, 214-502.

Hof, B., van Doorne, C. W. H., Westerweel, J., Nieuwstadt, F. T. M., Faisst, H., Eckhardt, B., Wedin, H., Kerswell, R. R. \& WalefFe, F. 2004 Experimental observation of nonlinear traveling waves in turbulent pipe flow. Science 305, 1594-1597.

Itano, T. \& ToH, S. 2001 The dynamics of bursting process in wall turbulence. J. Phys. Soc. Japan 70, 703.

Joseph, D. D. \& CARmi, S. 1969 Stability of Poiseuille flow in pipes, annuli, and channels. Q. Appl. Math. 26, 575-599.

KeRswell, R. R. 2005 Recent progress in understanding the transition to turbulence in a pipe. Nonlinearity 18, R17-R44.

Kerswell, R. R. \& TutTy, O. R. 2007 Recurrence of travelling waves in transitional pipe flow. J. Fluid Mech. 584, 69-102.

Leite, R. J. 1959 An experimental investigation of the stability of Poiseuille flow. J. Fluid Mech. 5, 81.

LeONARD, A. \& ReYNOLDS, W. C. 1985 Turbulence research by numerical simulation. In Perspectives in Fluid Mechanics: Proceedings of a Symposium Held on the Occasion of the 70th Birthday of Hans Wolfgang Liepmann (ed. D. Coles), Lecture Notes in Physics, vol. 320, p. 113. Springer.

Lindgren, E. R. 1958 The transition process and other phenomena in viscous flow. Ark. Phys. $12,1$.

LindgREN, E. R. 1969 Propagation velocity of turbulent slugs and streaks in transition pipe flow. Phys. Fluids 12, 418.

Mellibovsky, F. \& Meseguer, A. 2009 Critical threshold in pipe flow transition. Phil. Trans. R. Soc. A 367, 545-560.

Mellibovsky, F., Meseguer, A., Schneider, T. M. \& Eckhardt, B. 2009 Transition in localized pipe flow turbulence. Phys. Rev. Lett. 103, 054502.

Meseguer, A. \& Trefethen, L. N. 2003 Linearized pipe flow to Reynolds number 107 . J. Comp. Phys. 186, 178-197.

MoXey, D. \& BARKLeY, D. 2010 Distinct large-scale turbulent-laminar states in transitional pipe flow. PNAS 107, 8091-8096.

Mullin, T. 2011 Transition to turbulence in a pipe: a historical perspective. Annu. Rev. Fluid Mech. 43.

NAGATA, M. 1990 Three-dimensional finite-amplitude solutions in plane Couette flow: bifurcation from infinity. J. Fluid Mech. 217, 519-527.

Nishi, M., Unsal, B., Durst, F. \& Biswas, G. 2008 Laminar-to-turbulent transition of pipe flows through puffs and slugs. J. Fluid Mech. 614, 425-446.

Peixinho, J. \& Mullin, T. 2006 Decay of turbulence in pipe flow. Phys. Rev. Lett. 96, 094501.

Pfenniger, W. 1961 Transition in the inlet length of tubes at high Reynolds numbers. In Boundary Layer and Flow Control (ed. G. V. Lachman). NEW YORK: Pergamon.

Poiseuille, J. L. M. 1840 Recherches expérimentales sur le mouvement des liquides dans les tubes de très petits diamètres. C. R. Acad. Sci. 11, 961.

Pringle, C. C. T., Duguet, Y. \& Kerswell, R. R. 2009 Highly symmetric travelling waves in pipe flow. Phil. Trans. R. Soc. A 367, 457-472.

Pringle, C. C. T. \& Kerswell, R. R. 2007 Asymmetric, helical and mirror-symmetric travelling waves in pipe flow. Phys. Rev. Lett. 99, 074502.

Priymak, V. G. \& MiYazAKI, T. 2004 Direct numerical simulation of equilibrium spatially localized structures in pipe flow. Phys. Fluids 16, 4221-4234. 
Reuter, J. \& Dempfer, D. 2004 Analysis of pipe flow transition. Part 1. Direct numerical simulation. Theor. Comput. Fluid Dyn. 17, 273.

REYNOLDS, O. 1883 An experimental investigation of the circumstances which determine whether the motion of water shall be direct or sinuous and of the law of resistance in parallel channels. Phil. Trans. R. Soc. 174, 935-982.

SCHNEIDER, T. M. \& ECKHARDT, B. 2009 Edge states intermediate between laminar and turbulent dynamics in pipe flow. Phil. Trans. R. Soc. A 367, 577-587.

Schneider, T. M., ECKhardt, B. \& Vollmer, J. A. 2007a Statistical analysis of coherent structures in transitional pipe flow. Phys. Rev. E 75, 066313.

Schneider, T. M., EcKHARdt, B. \& Yorke, J. A. $2007 b$ Turbulence transition and the edge of chaos in pipe flow. Phys. Rev. Lett. 99, 034502.

Schneider, T. M., Gibson, J. F., Lagha, M., De Lillo, F. \& Eckhardt, B. 2008 Laminar-turbulent boundary in plane Couette flow. Phys. Rev. E 78, 037301.

SChNeider, T. M., Marinc, D. \& ECKhaRdT, B. 2010 Localized edge states nucleate turbulence in extended plane Couette cells. J. Fluid Mech. 646, 441-451.

Shan, H., Ma, B., Zhang, Z. \& Nieuwstadt, F. T. M. 1999 Direct numerical simulation of a puff and a slug in transitional cylindrical pipe flow. J. Fluid Mech. 387, 39-60.

Shimizu, M. \& KidA, S. 2008 Structure of a turbulent puff in pipe flow. J. Phys. Soc. Japan 77, 114401.

Shimizu, M. \& KidA, S. 2009 A driving mechanism of a turbulent puff in pipe flow. Fluid Dyn. Res. 41, 045501.

ToH, S. \& Itano, T. 1999 Low-dimensional dynamics embedded in a plane Poiseuille flow turbulence: traveling-wave solution is a saddle point? In Proceedings of IUTAM Symposium on Geometry and Statistics of Turbulence (ed. T. Kambe). Kluwer.

Viswanath, D. 2007 Recurrent motions within plane Couette turbulence. J. Fluid Mech., 580, 339-358.

Viswanath, D. 2009 The critical layer in pipe flow at high Reynolds number. Phil. Trans. R. Soc. 367, 561-576.

WalefFe, F. 1997 On the self-sustaining process in shear flows. Phys. Fluids 9, 883-900.

WaLefFe, F. 1998 Three-dimensional coherent states in plane shear flows. Phys. Rev. Lett. 81, $4140-4143$.

Wang, J., Gibson, J. \& WalefFe, F. 2007 Lower branch coherent states in shear flows: transition and control. Phys. Rev. Lett. 98, 204501.

Wedin, H. \& Kerswell, R. R. 2004 Exact coherent structures in pipe flow: travelling wave solutions. J. Fluid Mech. 508, 333-371.

Willis, A. P. \& Kerswell, R. R. 2007 Critical behavior in the relaminarization of localized turbulence in pipe flow. Phys. Rev. Lett. 98, 14501.

Willis, A. P. \& Kerswell, R. R. 2008 Coherent structures in local and global pipe turbulence. Phys. Rev. Lett. 100, 124-501.

Willis, A. P. \& Kerswell, R. R. 2009 Turbulent dynamics of pipe flow captured in a reduced model: puff relaminarization and localized 'edge' states. J. Fluid Mech. 619, 213-233.

Willis, A. P., Peixinho, J., Kerswell, R. R. \& Mullin, T. 2009 Experimental and theoretical progress in pipe flow transition. Phil. Trans. R. Soc. A 366, 2671-2684.

Wygnanski, I. J. \& Champagne, F. H. 1973 On transition in a pipe. Part 1. The origin of puffs and slugs and the flow in a turbulent slug. J. Fluid Mech. 59, 281-351. 